\title{
Picosecond-nanosecond laser flash, formation of powerful elastic waves in crystals, and shock peening
}

\author{
N. Inogamov \\ Landau Institute for Theoretical Physics of the Russian Academy of Sciences, 1-A \\ Akademika Semenova avenue, Chernogolovka, Moscow Region, 142432, Russian \\ Federation \\ Dukhov Research Institute of Automatics, 22 Sushchevskaya street, Moscow, 127055, \\ Russian Federation \\ Joint Institute for High Temperatures of the Russian Academy of Sciences, 13 \\ Izhorskaya street, Bld. 2, 125412 Moscow, Russian Federation \\ V. Zhakhovsky \\ Dukhov Research Institute of Automatics, 22 Sushchevskaya st., Moscow, 127055, \\ Russian Federation \\ Joint Institute for High Temperatures of the Russian Academy of Sciences, 13 \\ Izhorskaya street, Bld. 2, 125412 Moscow, Russian Federation \\ D. Ilnitsky \\ Dukhov Research Institute of Automatics, 22 Sushchevskaya st., Moscow, 127055, \\ Russian Federation \\ V. Khokhlov \\ Landau Institute for Theoretical Physics of the Russian Academy of Sciences, 1-A \\ Akademika Semenova avenue, Chernogolovka, Moscow Region, 142432, Russian \\ Federation \\ N. Inogamov: nailinogamov@gmail.com
}

Abstract Elastic-plastic transformations together or independently from polymorphic phase transitions are important for theory of shock waves. Here we discuss a classification consisting from (I) elastic, (II) split elastic-plastic, and (III) pure plastic shocks. The split shocks means that (1) there are two jumps: the elastic precursor and plastic shock, and that (2) the jumps are independent from each other, the precursor moves with elastic speed of sound overrunning the plastic jump and going further and further ahead as time proceeds and becoming weaker and weaker.

We oppose the split shock to the one-wave two zones shock (1W2Z shock). The 1W2Z wave (1) propagates as whole, (2) the plastic shock dynamically supports the elastic one, (3) the distance between the jumps does not change in time; here we say about the distance averaged over time.

The powerful elastic shocks (their amplitudes are much higher than are usually suggested for elastic shocks) were found in experiments with femtosecond laser pulses and confirmed in large scale molecular dynamics (MD) simulations. The observation of the $1 \mathrm{~W} 2 \mathrm{Z}$ shocks is another important finding coming from MD. In the paper below we want to emphasize that the $1 \mathrm{~W} 2 \mathrm{Z}$ wave (proved to exist in MD) is not some tiny submicron feature belonging to MD or to ultrashort shocks. We hypothesized that it may be detected at spatio-temporal scales significantly larger than micron. Thus it should be included into the above classification as a new regime. It should be placed between the II-nd and III-rd regimes in the old classification.

The report is devoted to lasers, shocks, and applications. In the second part of the report the generation and propagation of the shocks created for laser shock peening by lasers with ultrashort or nanosecond pulses are considered. 


\section{Introduction}

Studies of shock waves cover a very wide range of physical problems from hydrodynamics where the shocks complicate many flows (see, e.g., [1] - implosive collapse) to kinetic processes defining internal structure of a shock front (see, e.g., [2,3]; from collisionless shocks in rarefied hot plasma [4] to elastic-plastic and/or polymorphic transformations in solids $[2,5-15]$; shocks are accompanied by rarefaction waves causing spallation in condensed media $[10,12,13,16,17]$. Powerful laser shocks are used to explore equations of thermodynamic states of matter [18]. These stories are listed to show the base from which the report has been developed.

Elastic-plastic phenomena are important. They are closely connected with mechanics of deformable solids. Theories of creep and plastic motions often are formal, purely mathematical in nature. At the same time they are based on empirical measurements such as measurements of a bulk modulus $B$, a strength of materials or a yield stress $Y$.

Powerful and relatively new approaches for studies in this branch of sciences are connected with molecular dynamics (MD). These approaches allow us to see what is going on interatomic level, how the coordinated motions of ensembles of atoms takes forms of nucleating and evolving dislocations. It is not possible to achieve such resolutions in experiments, even using ultrashort (few femtosecond) hard x-rays for XRD in the pump-probe scheme following shock loading [17].

But molecular dynamics also has a significant drawback. Simulations are limited in their spatio-temporal sizes. Usually simulated condensed objects cannot be larger in volume than cubic micron and the duration of tracking of the evolution cannot be more than several tens of nanoseconds. G.I. Kanel' with coworkers systematically combines together data from small $(10-100 \mathrm{~nm})$ and large $(\mathrm{mm})$ scales obtaining universal dependencies, see Figures 4 and 5 in [6] for Hugoniot elastic limit and for spall strength, respectively. We see from that, that the small scale MD data are not exception but they are continuation of the well established mm-scale knowledge to the scales approaching to the ultimate theoretical strength - coupling between the mm-scale theories of plasticity and MD simulations.

In the previous classification (or ranging) there are three types of shock structures in solids: (I) an elastic shock, (II) a split elastic-plastic structure, and (III) a plastic shock. The structures change each other as piston pressure $p$ increases. A distance between the jumps increases in the case II - the plastic shock moves slower than the elastic shock, thus the elastic shock is called a precursor, a weak precursor ahead the stronger plastic one. Therefore we say about the split jumps.

Another regime of dynamic interaction between the elastic and plastic shocks was found in our MD simulations [8]. Let the plastic jump is continuously supported by a piston hold at permanent pressure $p$. Than thanks to interaction the plastic shock continuously supports the elastic front at a fixed distance $d$ ahead. Value of this distance depends on value of pressure $p$. We call this structure the one wave (because both jumps moves together at the same speed) two zone (because there are clearly defined elastic and plastic zones) wave - the 1W2Z shock for brevity.

Existence of the universal dependencies connecting atomic and macroscopic scales (they are mentioned above) lead us to find traces of the regime $1 \mathrm{~W} 2 \mathrm{Z}$ at large space-time dimensions. Before the existence of the $1 \mathrm{~W} 2 \mathrm{Z}$ propagation regime was confirmed up to the separation distance $d=200-400 \mathrm{~nm}[8]$. The separation $d(p)$ increases as pressure decreases. How large it may be? The first part of the paper is devoted to this problem.

In the second part the peculiarities of the shock formation, its propagation, and evolu- 
tion of solid transferred by the shock into a plastic state (evolution of solid after escape of a shock) are considered for subpicosecond $\left(\tau_{L} \sim 0.1 \cdot 10^{-12} \mathrm{~s}\right)$ and nanosecond $\left(\tau_{L} \sim 10^{-9}\right.$ s) pulse durations.

\section{Single entity: elastic compression as a first step of elastic- plastic transformation}

Nonlinear acoustic disturbance propagates with a Mach number larger than one. Hugoniot adiabatic curve starts from an initial point. Our initial point corresponds to uncompressed solid at room temperature. In an established (steady-state) state the disturbance generated by a piston supported by permanent pressure has to be a shock. The Hugoniot

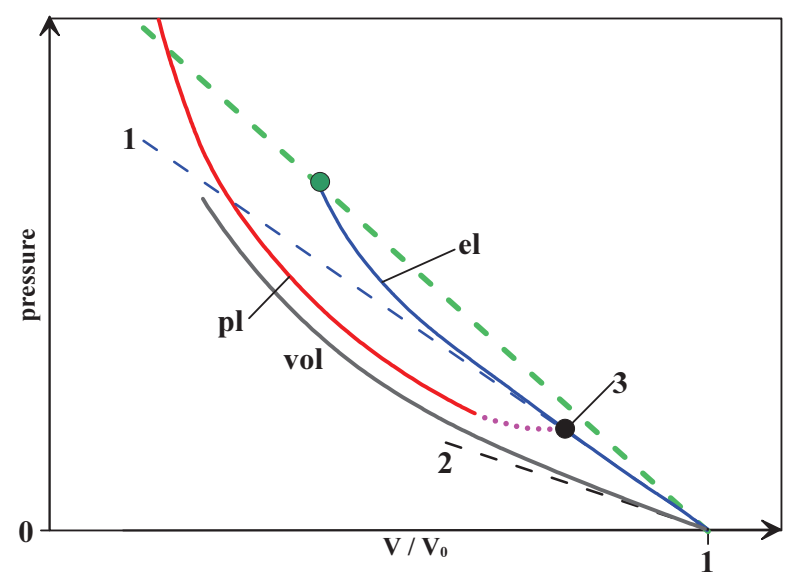

Fig.1 Comparison of hydrostatic (or volume, the curve "vol") and uniaxial compressions. "Volume" means homogeneous shrink along all three spatial axes. There are two types of the uniaxial compressions. One is the elastic ("el") while the second is plastic ("pl") compression. The "el" and "pl" curves relates to the corresponding Hugoniot curves. Elastic body is more rigid thus its Hugoniot is higher. Initial volume is $V_{0}$. Speeds of elastic and plastic sounds are the straights 1 and 2, respectively; approximation of isotropic elastic-plastic body is considered. The Hugoniot elastic limit is the black circle 3 .

curves are shown in Fig. 1.

The yield stress $Y$ elevates the plastic uniaxial compression above the hydrostatic one. This is because the initial elastic section is steeper than the plastic section. Difference between the curves "vol" and "pl" is (2/3) $Y$ according to the theory of elastic-plastic isotropic body. Plastic speed of sound $c_{p l}$ in the initial state defines the slope of the curve "vol" near initial point in Fig. 1 (the straight line 2). While the elastic Hugoniot adiabat "el" concerns the direct line 1 corresponding to the speed of elastic sound $c_{e l}=$ $\sqrt{[B+(4 / 3) G] / \rho}=\sqrt{c_{p l}^{2}+(4 / 3) G / \rho}=c_{p l} \sqrt{1+(4 / 3) G / B}$, where $B$ and $G$ are bulk and shear modulus. 


\subsection{Elastic shock: regime I}

Let's consider the classification of the structures of shocks. The regime I relates to a pure elastic shock. It occupies the range of piston pressures $p$ below the Hugoniot elastic limit: $0<p<p_{H E L}$. Corresponding Rayleigh line connects the initial point and the point belonging to the elastic Hugoniot curve "el" below the black circle 3 (see Fig. 1); we will call this circle the usual Hugoniot elastic limit (HEL).

Pressure corresponding to the circle $3 \mathrm{HEL}$ is small relative to the bulk modulus. Therefore the Hugoniot curve "el" in Fig. 1 is almost indistinguishable from the direct line 1 in this range of piston pressures. The regime I is simple, apart from the fact that the limit HEL is a conditional value. It is marked by the circle 3 in Fig. 1.

According to papers written by G.I. Kanel' with coworkers [6, 19-21] the value of HEL varies. For example, the value depends on the time (and vice versa) during which the uniaxial load $\sigma_{x x}$ is maintained in elastic solid. There is a definite waiting time $t$ for every value of the load $\sigma_{x x}$ when the elastic solid of fixed length transits to a plastic state: $t\left(\sigma_{x x}\right)$ and $\sigma_{x x}(t)$. There is connection between the value of HEL and the function $\sigma_{x x}(t)$. If $t$ is time of propagation of an elastic shock started in time $t=0$ and carrying the load $\sigma_{x x}(t)$ then we can write $x=c_{e l} t$ instead of $t$ in $\sigma_{x x}(x)$.

Considering the regime I we assume that the piston is driven by permanent pressure $p$ below the $p_{H E L}$ shown in Fig. 1 . The value $p_{H E L}$ gradually decreases with time as $\sigma_{x x}(x)$. Thus if we wait enough then the elastic shock passes distance $x=c_{e l} t$ and if the value of HEL $p_{H E L}(x)$ decreases below the piston pressure $p$ then our purely elastic shock (covering all space between a piston and a shock front) finishes and the flow transits from regime I to the regime II. After the transition a plastic shock begins to propagate from a piston behind the elastic shock.

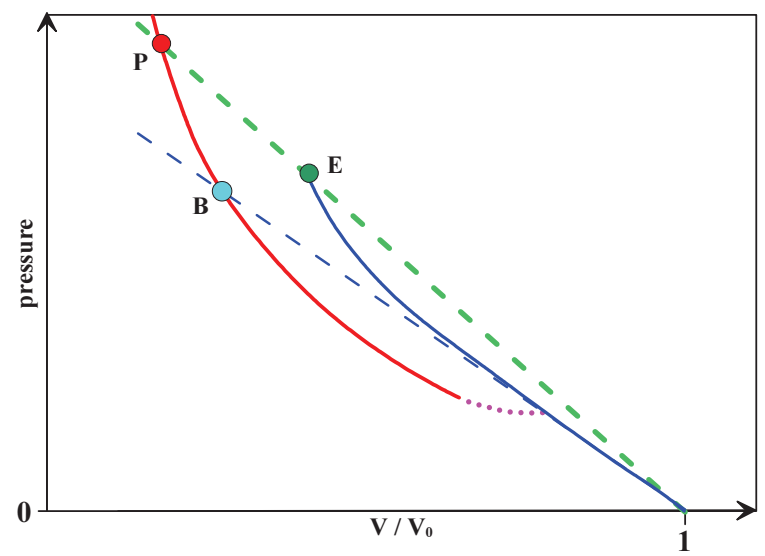

Fig.2 The elastic and plastic Hugoniots together with the two 1W2Z elastic plastic shocks. The shocks are presented by the Rayleigh lines 1-B and 1-E-P. The abbreviation 1W2Z means that there are two zones ("2Z": elastic 1-E and plastic E-P) in the internal structure of the single wave. The wave is single ("1W") because it moves as whole: both segments 1-E and E-P have equal slopes since they belong to the single Rayleigh line. As it is known, the slope defines propagation velocity. 


\subsection{A family of the single wave elastic-plastic shocks}

As was said above, the boundary $p_{H E L}$ of the pressure $p$ on the piston between the elastic shock regime I and the split elastic-plastic regime II is not strictly defined. We better understand the meaning of the split regime II, if we first consider the $1 \mathrm{~W} 2 \mathrm{Z}$ regime. The examples of the $1 \mathrm{~W} 2 \mathrm{Z}$ regimes are shown in Fig. 2.

The $1 \mathrm{~W} 2 \mathrm{Z}$ shocks form an one-parametric family. The chosen $1 \mathrm{~W} 2 \mathrm{Z}$ shock in the family is defined or by its particular propagation velocity, or by its piston pressure $p$. The separation distance $d(p)$ between the elastic and plastic shocks inside the particular structure depends on pressure $p$. The distance decreases as the pressure $p$ increases.

The upper limiting $1 \mathrm{~W} 2 \mathrm{Z}$ wave corresponds to the upper value of the pressure $p_{u p}$. At this pressure the distance $d$ becomes comparable to the interatomic distance. Thus for the even higher pressures $p>p_{u p}$ only the pure plastic regime exists. For aluminum the values $p_{u p}$ are somewhere in the range $40-65 \mathrm{GPa}$ depending on the state of metal inflowing into a shock structure [8].

\subsection{The weakest $1 \mathrm{~W} 2 \mathrm{Z}$ wave}

In Fig. 2 the family of the $1 \mathrm{~W} 2 \mathrm{Z}$ waves is shown. The upper edge of this family is easily defined, see subsection 2.2. How to define the bottom edge 1-B in Fig. 2?

Let's calculate velocity of a plastic shock from Hugoniot adiabatic curve (from the Rankine-Hugoniot relations which follow from conservation laws). It is known that up to very strong shocks the shock Hugoniot is well approximated by the so called "us-up" relation. For a plastic shock this relation is

$$
u_{s}=c_{p l}+a_{p l} u_{p}
$$

where $u_{s}$ is shock velocity, $a_{p l}$ is dimensionless coefficient $\sim 1$, and $u_{p}$ is velocity of a piston in the frame where material is at rest ahead to the shock front. Material comes to a shock with velocity $u_{s}$ and passes in with velocity $u_{s}-u_{p}$ in the reference frame propagating together with a front.

For aluminum the coefficients $(1)$ are $c_{p l}=5.35 \mathrm{~km} / \mathrm{s}, a_{p l}=1.37$, see e.g. $[7,8]$. Velocity of a plastic shock $\left.\left.u_{s}\right|_{p l}\right|_{\text {crit }}$ corresponding to the $1 \mathrm{~W} 2 \mathrm{Z}$ wave limiting the family from the bottom side is equal to the elastic speed of sound:

$$
\left.\left.u_{s}\right|_{p l}\right|_{c r i t}=c_{e l} \text {. }
$$

The condition (2) corresponds to the Rayleigh line 1-B in Fig. 2.

For aluminum an elastic speed of sound $c_{e l}$ is $\approx 6.5 \mathrm{~km} / \mathrm{s}$. Then from equation (1) we obtain

$$
\left.\left.u_{p}\right|_{p l}\right|_{c r i t}=\left(c_{e l}-c_{p l}\right) / a_{p l}=0.84 \mathrm{~km} / \mathrm{s} .
$$

This is the slowest piston still supporting the weakest $1 \mathrm{~W} 2 \mathrm{Z}$ wave.

Knowing piston velocity (3) we can find corresponding compression $\left.\left.\left(V / V_{0}\right)\right|_{p l}\right|_{\text {crit }}$ behind the plastic shock. The expression for compression follows from kinematic conditions and conservation of mass $\rho_{0} u_{s}=\rho\left(u_{s}-u_{p}\right)$. The expression for the critical compression is

$$
\left.\left.\left(V / V_{0}\right)\right|_{p l}\right|_{c r i t}=1-u /\left(c_{p l}+a_{p l} u\right)=0.87
$$

where $u$ is $\left.\left.u_{p}\right|_{p l}\right|_{\text {crit }}(3)$. 
Corresponding critical pressure is defined by the value (4) and the momentum conservation law. This pressure is

$$
p_{c r i t}=\rho_{0} c_{p l}^{2} \frac{1-V / V_{0}}{\left[1-a_{p l}\left(1-V / V_{0}\right)\right]^{2}}=14.9 \mathrm{GPa},
$$

where $V / V_{0}$ is given by the expression (4). The pressure (5) is the pressure at a piston in the point $\mathrm{B}$ in Fig. 2. This is the pressure in a plastically compressed aluminum behind a plastic front. Previously the point $(3,4,5)$ was called the overdriven point.

It is difficult to estimate the distance $d$ between the jumps for the plastic compression (4) and plastic pressure (5). But we suggest that it is very significantly larger than the micron scales achieved in MD simulations [8]. Indeed, according to Fig. 2 for the bottom end of the one-parametric family with the Rayleigh line 1-B the pressure behind the elastic shock is somewhere near the traditional values for the Hugoniot elastic limit. That is, it is small, less than $1 \mathrm{GPa}$ for aluminum. Then a shear stress behind such elastic shock uniaxially compressing aluminum is rather small. Then a degree of metastability is small. This means that aluminum may survive in such states much longer than in MD simulations [8].

\subsection{The split regime}

The split regime obviously appears for piston pressures below the limiting value (5). Velocity of a plastic front decreases below the elastic speed of sound. Thus a plastic shock begins to systematically leave the elastic one.

If an elastic wave is removed a long distance from the plastic one, then the elastic wave loses dynamic support from the plastic shock. The plastic shock is permanently driven by a constant piston pressure. Therefore a pressure field behind the plastic front is homogeneous.

While the elastic front escaping from support decays thank to its nonlinear stretching. The elastic shock transits to a triangular shape. The triangular becomes wider and wider because of weak but finite nonlinear effects: dependence of speed of sound on amplitude dispersive weakening. This is a picture with asymptotically dynamically unrelated objects: plastic and elastic fronts.

Although usually a different picture is drawn. In the usual picture there are two Rayleigh lines with different slopes. The kink between these lines is supported by the point 3 (traditional Hugoniot elastic limit) in Fig. 1 above, see e.g. [23]. But slowly the point 3 decreases along the elastic Hugoniot. Thus, outwardly, both pictures become similar.

While in the $1 \mathrm{~W} 2 \mathrm{Z}$ case the elastic and plastic shocks are dynamically connected. Their separation distance $d$ fluctuates near the average value $d(p)$ depending on a piston pressure. There are two types of disturbances causing the fluctuations. In the first type the delay of elastic-plastic transformation near the plastic front pushes elastic front because untransformed elastic metal have larger volume than transformed one. The delayed transition sends a triangular elastic waves propagating from plastic to elastic front [8]. Flow behind an elastic shock is subsonic relative to elastic speed of sound. Therefore, the triangular waves catch up with the elastic front and throw it forward.

In the second variant of the dynamic connection, the column of uniaxially elastically compressed crystal from time to time experiences collapses [22,23]. The collapse shrinks length of the column thanks to additional compression of metal around the nucleus of 
destruction. The places and moments when a specific collapse begins are controlled by a probabilistic process. Usually they take place more close to the plastic shock. In this case the piece of the elastically compressed crystal is longer hold under load. Two rarefaction run away from the place of collapse. This process decreases slightly too high longitudinal stress at the elastic front thus adjusting an elastic load to corresponding piston pressure.

The picture of the transverse interaction of points of collapse is extremely interesting $[22,23]$. Three dimensional effects are also very beautiful in the first variant with elasticplastic transformation near the plastic front $[8,14,15]$.

The proposed classification of the shock structures is: (I) simple pure elastic shocks; (II) the split regimes; (III) 1W2Z regimes; and (IV) simple pure plastic shocks.

We may say that the regime II is the transition regime to regime IV. But the transition takes an infinite amount of time - we have to wait for the elastic precursor to go to infinity from the plastic front.

\section{$3 \quad$ Peculiarities of picosecond laser loading of solids}

Often a laser is used as a tool to create a load in a solid; let's mention in this connection, e.g., using of lasers for studies of equation of state [18]. But this tool has its own very specific features. The tool causes heating of an absorbing surface layer. Thickness of a heated layer $d_{T}$ is defined by a light penetration depth in an absorbing matter (a skin-layer in metals for optics) and thermal conductivity.

Strong load means intense heating. Thus melting and evaporation phenomena become significant. The characteristics associated with melting and evaporation are well known. There is an additional feature linked specially to lasers with ultrashort pulse. This is a pronounced mechanical action of such a pulse.

Let's define the term "ultrashort pulse". The pulse is ultrashort if its duration $\tau_{L}$ is less than acoustic time scale $t_{s}=d_{T} / c_{s}$. Then the hydrodynamic unloading is delayed relative to the duration of the period of time $\tau_{L}$ for which the heating takes place. After such laser pulse matter unloads under pressure $p \approx \Gamma E$, where $\Gamma \approx 2$ is Gruneisen parameter, $E \sim F_{a b s} / d_{T}$ is volume density of absorbed energy, $F_{a b s}$ is absorbed fluence. For example, $d_{T} \sim 100 \mathrm{~nm}, t_{s} \sim 20 \mathrm{ps}, F_{a b s} \sim 0.3 \mathrm{~J} / \mathrm{cm}^{2}, p \sim 60 \mathrm{GPa}$.

A laser pulses of longer durations $\tau_{L}$ also drive pressure, but less effectively: $p \sim$ $\left(t_{s} / \tau_{L}\right) \Gamma E$, see next section.

Ultrashort pulse creates significant tensile stress. This is the main qualitative difference from the action of long pulses. Thus, long pulses can melt and evaporate the target material. While an ultrashort pulse can mechanically tear off a layer of matter in the irradiation spot.

This phenomenon is called spallation or cavitation. It explains formation of the Newton rings $[24,25]$. Spallation develops after nucleation of voids $[10,12,13,16]$. The nucleation begins above the nucleation threshold $\left.F_{a b s}\right|_{n u c l}$. For ultrashort laser pulse the nucleation and spallation take place in liquid phase - a surface layer melts before the nucleation.

Surface tension resists to expansion of voids. Therefore the spallation (or ablation) threshold $\left.F_{a b s}\right|_{a b l}$ is higher than the nucleation threshold $\left.F_{a b s}\right|_{n u c l}[26,27]$. Capillary forces may be very significant increasing the threshold $\left.F_{a b s}\right|_{a b l}$ approximately twice above the nucleation threshold $\left.F_{\text {abs }}\right|_{\text {nucl }}$. Such large increase is observed in metals like Mo, Ta, Ni with a high coefficient of surface tension and thin heated layers $d_{T}$; thermal conductivity is small. Inertia of a thin layer is small thus surface tension is more effective in deceleration 
of these layers.

A prominent rim surrounds the laser spot [27]. Its position corresponds to the intermediate range of absorbed energies $\left.F_{a b s}\right|_{n u c l}<F_{a b s}<\left.F_{a b s}\right|_{a b l}$. In this range a nucleation takes place but the surface layer retains its mechanical connection with the target. Thus a porous surface layer appears [27].

Surface structures appear due to competition between inertia/capillary dynamics and cooling/freezing thermal processes. Subtle effects take place depending on the relationship between mechanical and thermal factors. Common shell covers the laser spot. The shell is in motion. For rather large spots (few tens of microns) the shell at an initial stage is composed from many individual shells covering their particular expanding bubble (the bubble is a product of expansion of the individual nucleus). Every individual shell is separated from its neighbors by a membrane.

If freezing is rather slow, then the separation membranes have time to break. Then the well defined wall is formed around the spot [28]. But in the opposite case, with high thermal conductivity and a thin liquid layer under the bottoms of the bubbles, the separation membranes remain frozen. These frozen membranes form a random edge of a spot.

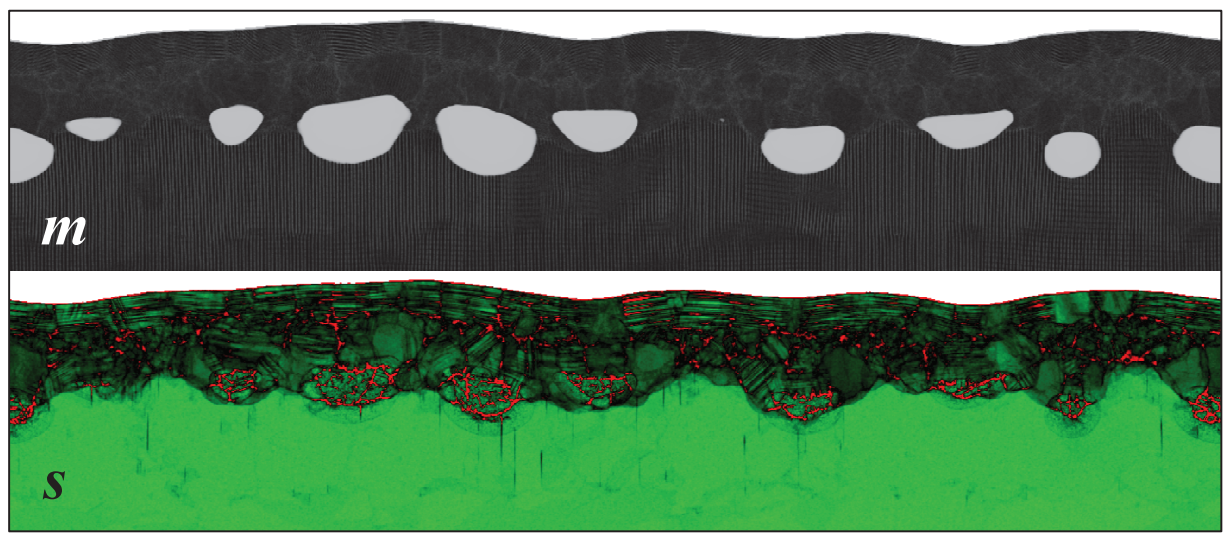

Fig.3 Frozen surface nano-structures remaining in a thin layer near surface after action of a picosecond laser pulse are shown. The upper panel "m" gives density distribution. The bottom panel "S" demonstrates distribution of a symmetry factor S. The factor shows local order of a crystallin lattice. Green colors corresponds to a solid state. Regions colored with deep green shows nano-crystalline aluminum. We are interested in the residual deformations produced in a target by an ultrashort laser pulse. From this point of view, melting, nucleation and freezing of nano-cavities is a side effect. The horizontal dimension is 0.5 micron.

\subsection{Formation of frozen nanocavities and residual stresses}

Fig. 3 presents the final structures located near frontal free surface; laser acts to the frontal surface. Final means that this picture corresponds to late time after finishing of a pulse. At that late stage the structures are totally crystallized. Cavities are frozen into 
solid surrounding, compare with simulations and experiments in [27]. Pulse duration is $\tau_{L}=1$ ps. The time shown in Fig. 3 is 0.6 ns.

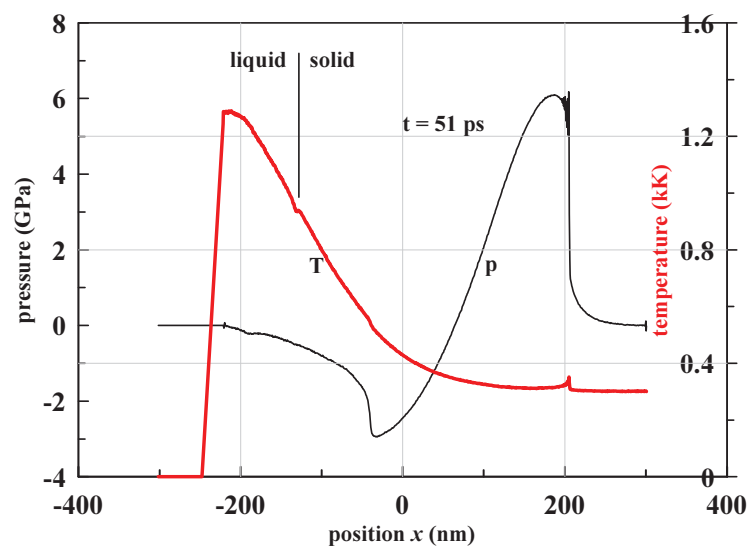

Fig.4 Distributions of pressure and temperature in the longitudinal direction at the early stage. At this stage the shock still is approaching the rear-side of a film. The distributions are obtained after averaging along the transverse directions relative to the vertical (longitudinal) direction. Laser beam comes from the left side.

Aluminum film with dimensions $500 \times 500 \times 24 \mathrm{~nm}^{3}$ was considered in this MD-MC simulation; MD means molecular dynamics, MC is Monte-Carlo subroutine describing electron thermal conductivity of aluminum. Electron thermal conductivity is high. It cools down a hot surface layer. Therefore the liquid phase freezes. In simulation shown in Figures 3-6 357 million atoms was used. The simulation box is bounded by periodic boundary conditions in the transverse directions. Absorbed fluence of $70 \mathrm{~mJ} / \mathrm{cm}^{2}$ is below the ablation threshold $\left.F_{a b s}\right|_{a b l}$ but above the nucleation threshold $\left.F_{a b s}\right|_{\text {nucl }}$.

Residual dislocations are clearly visible under the layer of frozen bubbles in Fig. 3. They are formed by combined action of shock compression and thermomechanical stress field. Deep green colors around the frozen bubbles show the nano-crystallin aluminum.

Distributions of pressure and temperature in the longitudinal direction at the early stage is shown in Fig. 4. We see the shock propagating from left to right. The shock is formed after breaking (turnover) of a compression wave created by supersonic heating. We call the heating supersonic because duration of a pulse $\tau_{L}=1$ ps is much less than acoustic time scale $t_{s}=d_{T} / c_{s} \approx 20$ ps. This is connected with superfast expansion of absorbed heat from a skin-layer to the heat affected zone $d_{T} \approx 100-120 \mathrm{~nm}$. Just few picosecond $t_{T}$ are necessary to create the layer $d_{T}$. The speed $d_{T} / t_{T}$ of expansion of internal energy during forming the layer $d_{T}$ is $\approx 7-10$ times higher than speed of sound in aluminum.

Reflection of a compression wave from a free surface produces a back part of a wave. This part is carrying a tensile stress inside a target, see Fig. 4. The tensile part has a trace of a spallation shock. This shock forms when molten aluminum begins to nucleate. The liquid layer is shown in Fig. 4. The deep well in the density profile shown in Fig. 5 is the consequence of formation of bubbles.

Pressures are moderate, but significantly below a bulk modulus of aluminum. There- 


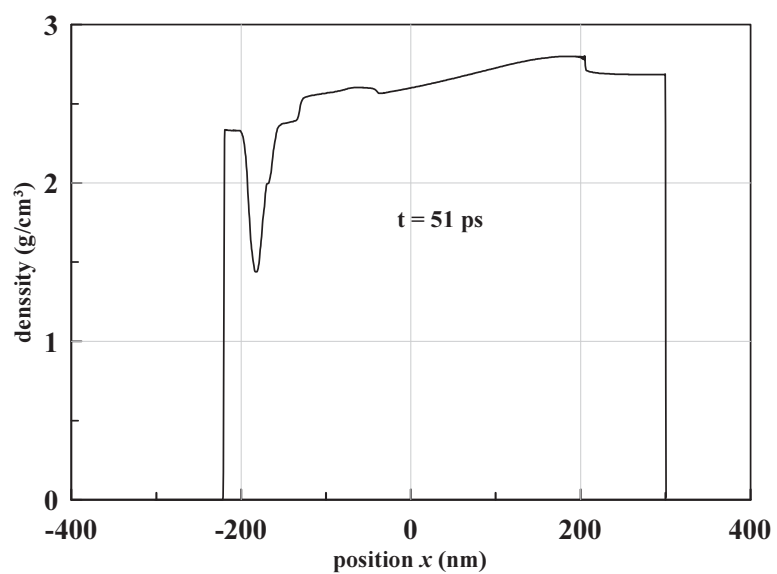

Fig.5 Density distribution along the longitudinal direction. This is the vertical direction in Fig. 3. The deep well at the left side near the free surface corresponds to the chain of bubbles. These bubbles nucleate due to action of the tensile part of the pressure profile shown in Fig. 4.

fore compressions and expansions related to the compression and tensile part of pressure profile in Fig. 4 are weak at the density profile shown in Fig. 5. The shock weakly heats aluminum - see small temperature jump corresponding to the shock in Fig. 4.

Final state of aluminum is shown in Figures 3 and 6 . We construct special boundary condition at the rear side of the film. This is the right side seen in Figures $4-6$. This condition leaves the shock seen in Fig. 4 through the right boundary without reflection.

Thus our simulation describes the case of an infinite target. In this target the acoustic profile shown in Fig. 4 travels to the bulk and loses its connection to the surface layer.

Aluminum in Figures 3 and 6 cools down to temperature $400 \mathrm{~K}$. This temperature is near the initial (prior to laser heating) temperature $300 \mathrm{~K}$. Temperature gradient across the film is small, approximately $70 \mathrm{~K}$.

We want to emphasize two remarkable features.

The first is the frozen undersurface nano-bubbles surrounded by solid nano-crystallin matrix.

The second feature relates to the residual strain and stress fields. We clearly see the remaining fields in Figures 3 and 6. They correspond to late enough time - the 30 acoustic time scales $t_{s}=20 \mathrm{ps}$.

Longitudinal stress is near to zero - unloading due to presence of a free surface. But the transverse stresses survive at the level of few hundred MPa. A thin stressed layer is located near the bubbles, compare the density profile and the profiles of transverse stress in Fig. 6. It seems, that this layer is connected with thermal processes around the bubbles. The deeper layer seems is formed due to plastic transformations under action of compression. 


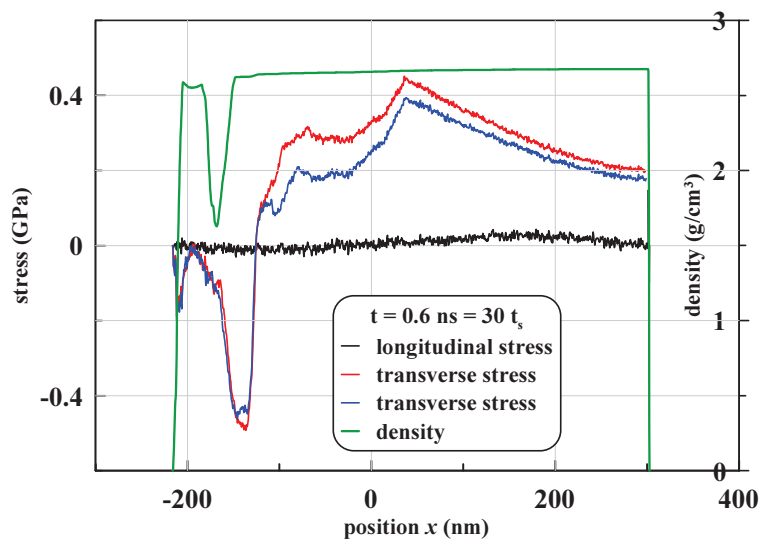

Fig.6 Final profiles are shown. They correspond to the late instant 0.6 ns. Density and symmetry maps for this time are presented in Fig. 3. We see creation of significant residual stress field. It relates to the residual deformations shown in Fig. 3.

\section{Laser shock peening by nanosecond laser pulse}

Laser action has long been used to generate shock waves in condensed matter. The traditional branch of these applications is to clarify the equations of state (EOS) at high energy densities that are unattainable with chemical explosives [18]. The other branch is connected with the modern technology of laser peening. With the help of peening significantly (tens, hundreds of percent [29]) increase resistance to wear (fatigue failure) and resistance to corrosion. For example, strengthen the details of aircraft engines https : //en.wikipedia.org/wiki/LSP/Technologies, https : //www.lsptechnologies.com/history.php

In this paper, we want to draw attention to significant common phenomena for such different technologies as laser peening and ablation in liquid. At the same time, the relevant scientific communities are largely disconnected. Similar experiences are interpreted differently. In peening experiments, crater formation is associated with residual plastic deformations (ablation is neglected), see, for example, [30]. Whereas in experiments with ablation, it is believed that a crater is formed due to the entrainment of a substance into a liquid [31], i.e. the mechanics of the deformation of a solid below the surface of the crater are neglected. Meanwhile the pulse duration of the nanosecond, and the intensity comparable $1-10 \mathrm{GW} / \mathrm{cm}^{2}$ in both technologies; to enhance the effect usually work in the area of the optical breakdown of the liquid. The aluminum target was used in [30], while [31] considered a corundum target; the deformation of brittle materials, which include corundum, is difficult [32].

Of course, understanding the physics of the phenomena taking place is important for successful work. In many ways, this understanding is based on analytical solutions that are complemented by numerical calculations. In the introductory part of this paper, we turn our attention to analytics related to the ablation into water and peening problems. These analytical solutions describe the expansion into vacuum and condensed matter, long and short pulses. Below is a short list of solutions. In the 70-90s of the XX-th century,a detailed analytical theory of the plasma laser corona was developed in the approximation 
of a stationary flow and a fixed degree of ionization [33,34]. This solution considers the expansion of the substance in the corona into a vacuum; the energy absorbed in the region of critical density is transmitted by the electronic thermal conductivity to the ablation front, which moves along a relatively cold dense substance.

In the case of ultrashort laser pulses (USLP, there is no transition to a stationary flow) and/or during the expansion into a condensed (not vacuum and not gas) environment, the analysis, of course, changes qualitatively (compared to the plasma corona). A simple thermo-acoustic solutions to help the understanding and implementation of the first estimates, for the case of the impact of USLP on thin films is given in [35]; thin film is considered, the laser warm-up time of which $t_{T} \sim d_{f}^{2} / 4 \chi$ throughout the thickness is less that the time $t_{s}=d_{f} / c_{s}$, for which the sound runs through the thickness; duration of $\tau_{L}$ of the pulse is small $\tau_{L}<t_{T}$; here and below $d_{f}$ is the film thickness, $\chi$ is the thermal diffusivity of the film, $c_{s}$ is the speed of sound.

The analytical approach in the case of USLP and ablation into the liquid [36] is based on the classical solution of the decay of the gas-dynamic gap: at $t<0$ we have two halfspaces in contact with each other, at $t=0$ the separating wall is removed. Substances in half-spaces have different pressure values and at $t<0$ they rest. In the case of long pulses $\tau_{L} \gg d_{T} / c_{s}$, the analyst of heating of an absorbing target through a transparent dielectric is completely different [37], here $d_{T}$ is the thickness of the heating layer. Figure 7 presents the corresponding scheme. Below we describe this scheme in detail and compare it with the results of our numerical calculations.

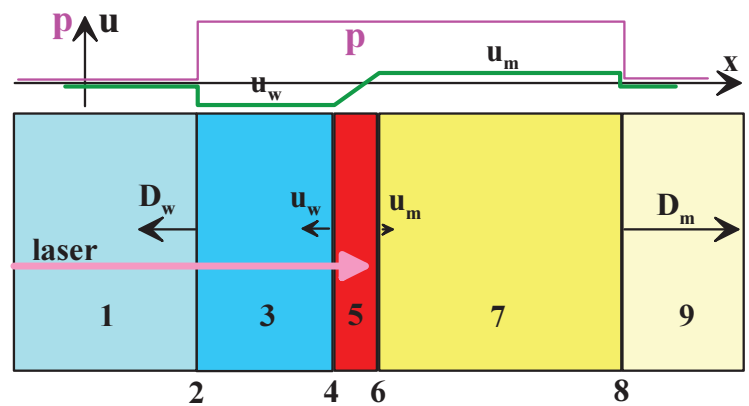

Fig.7 Heating metal through water-arrow "laser". The model [37] is presented. The laser passes through unperturbed water 1 , crosses the shock wave front 2, passes through the shock-compressed water 3 and is absorbed in the hot layer 5 . The pulse is long in time ("subsonic" heating) in the sense that the sound scale $L / c_{s}$ is much shorter than the pulse duration $\tau_{L}$, here $L$ is the width of the layer 5 . Therefore, the boundaries 4 and 6 of layer 5 act as two subsonic pistons pushing waves 2 and 8 . Waves 2 and 8 propagate much faster than the expansion rate of layer 5 . Therefore, layer 5 is thin compared with layers 3 and 7 .

\section{On the heating layer supporting nonlinear acoustic waves.}

Figure 7, taken from [37], shows a wide area of acoustic perturbation from $x_{2}$ to $x_{8}$. It is created by a long-lasting laser pulse. The width of $x_{8}-x_{2}$ is approximately $\left(c_{w}+c_{m}\right)(t-$ $\left.t_{\text {ini }}\right)$. The time $t$ will be counted from the maximum of the laser pulse with a Gaussian 
profile $I=I_{0} \exp \left(-t^{2} / \tau_{L}^{2}\right)$. The moment of the pulse in numerical calculation denote $t_{\text {ini }}$; we have $t_{\text {ini }}=-3 \tau_{L}$.

Sound velocities in water and metal are $c_{w}$ and $c_{m}$. For absorbable intensities $I_{\text {abs }} \sim$ $1 \mathrm{GW} / \mathrm{cm}^{2}$ and durations $\tau_{L} \sim 1 \mathrm{~ns}$ the pressure to the end of the pulse of the order of $1 \mathrm{GPa}$. These pressures are small compared to the bulk metal module $B_{m} \sim 10^{2} \mathrm{GPa}$ and of the order of the water module $B_{w}=1.5 \mathrm{GPa}$. Therefore, the acoustic perturbation running through the water in figure 7 , is moderately nonlinear and weakly nonlinear in metal.

The pistons 4 and 6 create a simple Riemann waves, respectively, in water and metal. Focusing of characteristics and wave breaking of the Riemann is happening in the water early due to the high nonlinearity of the waves in the water. In the linear acoustic approximation, the pressure and velocity are connected via the acoustic impedance $p=$ $z u$. In the model [37], the pressure is uniform over the interval from $x_{2}$ to $x_{8}$, see the graph in the upper part of figure 7 . Then the water velocities $u_{w}$ and metal $u_{m}$ are homogeneous, each in its own domain, i.e. in 3 and 7 . The expansion rate of the hot layer is $\dot{L}=\left(1 / z_{w}+1 / z_{m}\right) p$. In the numerical calculations below, we consider a pair of gold and water. Acoustic impedance of water is small: $z_{w} l_{m}$. So $\dot{L} \approx p / z_{w}$.

The above relation $\dot{L}=p / z_{w}$ follows from the acoustic approximation. A difficult place is to establish a balance of energy. Energy supply to the hot layer 5 (arrow "laser" in figure 7), firstly, heats the substance of the layer and, secondly, transfers energy to acoustic waves in water and metal. In [37] this balance is written as:

$$
I-p \dot{L}=(\mathrm{d} / \mathrm{dt})\left(E_{\text {int }} L\right)
$$

That is, the intensity absorbed in the layer minus the mechanical work of the pistons is spent on increasing the internal energy $E_{\text {int }}$ of layer 5 . In this case, it is assumed that new portions of the substance do not enter layer 5; i.e., layer 5 is created at an early stage, and then its mass does not change. It is necessary to link the energy $E_{\text {int }}$ and the pressure $p$, generated by heating in layer 5 . Then the equation (6) will allow us to express the pressure $p$ through the intensity $I$.

In the model [37], the following assumptions are made. The internal energy of $E_{\text {int }}$ is divided into two terms: $E_{\text {int }}=E_{T}+E_{\text {ion }}$, where $E_{T}=\alpha E_{\text {int }}$ is the thermal energy that determines the pressure of $p$, and $E_{\text {ion }}=(1-\alpha) E_{\text {int }}$ is the energy that is spent on the ionization of the substance in layer 5 and is not involved in the pressure increase $p$. Thus, the effective increase of heat capacity during ionization is taken into account. It is assumed that the coefficient $\alpha$ is constant. In addition, it is assumed that $p=(2 / 3) E_{T}$, as in the case of a monatomic ideal gas. Under these assumptions, for a constant laser pulse we obtain:

$$
\begin{aligned}
p & =\sqrt{\frac{2 \alpha}{2 \alpha+3} z_{w} I}=5.5 \sqrt{\frac{\alpha}{2 \alpha+3}} \sqrt{\frac{I}{1 \mathrm{GW} / \mathrm{cm}^{2}}} \mathrm{GPa}, \\
u_{w} & =p / z_{w}=3.7 \sqrt{\frac{\alpha}{2 \alpha+3}} \sqrt{\frac{I}{1 \mathrm{GW} / \mathrm{cm}^{2}} \mathrm{~km} / \mathrm{s} .}
\end{aligned}
$$

Here $z_{w}$ is the water impedance; in numerical estimates $(7,8)$ the value $z_{w}=1 \mathrm{~km} / \mathrm{s}=$ $1 \mu \mathrm{m} / \mathrm{ns}$ is substituted, so during the time of the order of $\tau_{L} \sim 1$ ns the hot layer will expand to the thickness $L \sim 1 \mu \mathrm{m}$. According to [37], the coefficient $\alpha$ is small $(\sim 0.1)$, and therefore significant. 


\section{Heating, expansion and acoustic radiation: the role of melt- ing.}

We present the results of numerical simulation of the problem described by the approximate analytical model in the section above. Consider a gold-water pair. Laser radiation passes through water and is absorbed in gold. The corresponding code was described in $[35,36]$, see also [38-42]. The pulse shape has the form $I=I_{0} \exp \left(-t^{2} / \tau_{L}^{2}\right), \tau_{L}=0.5 \mathrm{~ns}$, the absorbed energy is $0.9 \mathrm{~J} / \mathrm{cm}^{2}$.

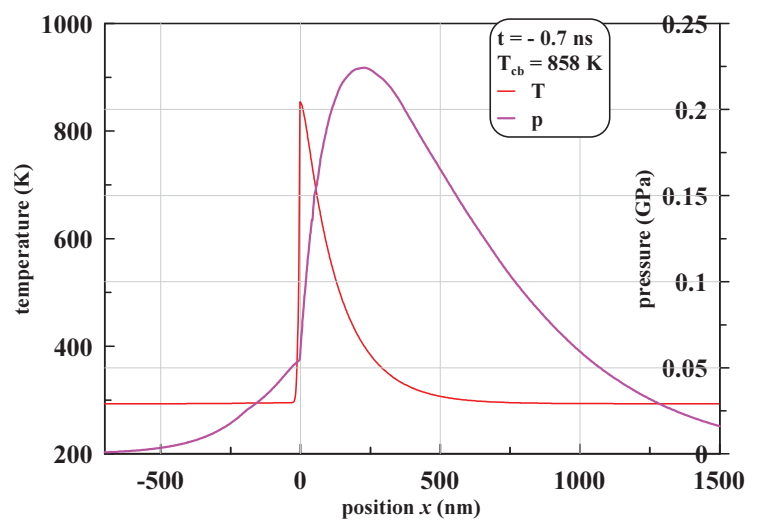

Fig.8 The situation at stage I, i.e. before melting, is shown. The calculation is started at $t_{\text {ini }}=-1.5 \mathrm{~ns}$. At this point, gold is to the right of the plane $x=0$, and water is to the left. The initial temperature of water and gold $T_{\mathrm{rt}}=300 \mathrm{~K}$. The heat spreads to gold at a depth of $d_{T}=0.1 \mu \mathrm{m}$. In the plane at a depth of $d_{T}$ the temperature is $\left(T_{\max }-T_{\mathrm{rt}}\right) / 2+T_{\mathrm{rt}}=\left(T_{\max }+T_{\mathrm{rt}}\right) / 2$, i.e. $d_{T}=0.1 \mu \mathrm{m}$ is the full width at half-height by temperature increase. The pressure profile has smooth acoustic wings on the left and right.

The flow in its development goes through a number of stages. The first is stage I, where the solid metal is heated. This stage ends when the melting of the metal begins. This is followed by stage II, in which the melt layer expands, and its temperature on contact with water increases. At the end of stage II, the temperature of the metal on the contact reaches a critical temperature of gold. In stage III, a gaseous layer of metal is formed near the contact. Stage IV refers to the end of the heating pulse. At this stage, a deep dip in the pressure profile begins to form. This dip is in the contact area. The appearance of the dip is due to the termination of heating of the contact area and the divergence in the direction of the acoustic perturbation formed during the action of the laser pulse.

Let us illustrate the above with respect to the stages of the process. Let's start with stage I and its transition to stage II. The figure 8 shows the instantaneous temperature and pressure profiles shortly before the end of the stage I - the laser heats the metal, but the metal temperature remains below the melting temperature, for gold $T_{\mathrm{m}}=1337 \mathrm{~K}$. The thickness of the heated layer of metal $d_{T}=0.1 \mu \mathrm{m}$. If we estimate this thickness by the formula $2 \sqrt{\chi\left(t_{\mathrm{obs}}-t_{\mathrm{ini}}\right)}$, we get about $0.7 \mu \mathrm{m}$; here $\chi \approx 1.2 \mathrm{~cm}^{2} / \mathrm{c}$ is the temperature conductivity of solid gold, $t_{\mathrm{obs}}=-0.7$ is the time point at which the graphs in figure 8 , 


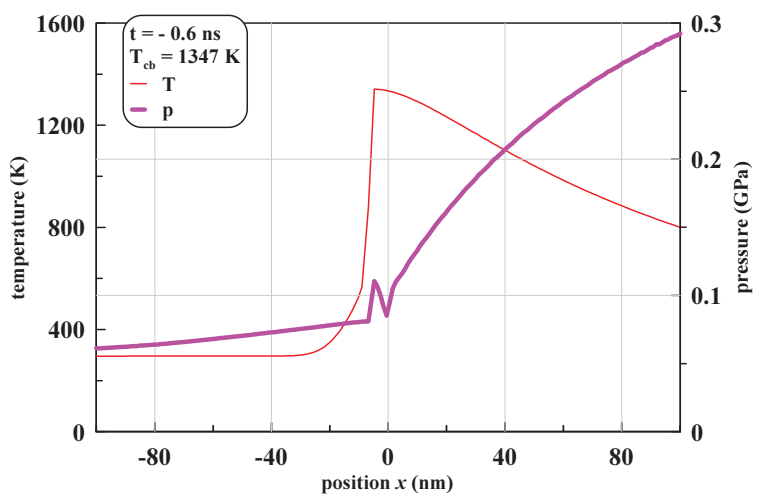

Fig.9 Beginning of stage II with a layer of melt. The contact temperature $T_{\mathrm{cb}}=1347 \mathrm{~K}$ by 5 degrees surpassed the melting point $T_{\mathrm{m}}(p)$ of gold under a pressure of 943 bar in the region of the contact boundary $(\mathrm{cb})$. The dependence of $T_{\mathrm{m}}(p)$ is shown in the following two figures. At the moment of formation of the liquid phase, a tooth is formed on the pressure profile $p$ in the contact area. At stage I, the $p$ profile is smooth everywhere - there are no teeth anywhere.

$t_{\text {ini }}=-1.5 \mathrm{~ns}$ - the time when the code starts to work. It is clear that the estimate of $2 \sqrt{\chi \Delta t}$ does not work, because the wing of the time intensity distribution $I \propto \exp \left(-t^{2} / \tau_{L}^{2}\right)$ is very weak in the time interval near the moment of the beginning of the calculation $t_{\text {ini }}$. Indeed, if $t_{\text {ini }}$ is carried away to minus infinity, then this estimate will give an infinite thickness of the heated region, whereas the profiles in figure 8 practically will not change.

Pressure profile in figure 8 has smooth spatial wings on the left in water and on the right in gold. These are acoustic perturbations associated with the time-axis left wing of the time profile of the laser pulse $I \propto \exp \left(-t^{2} / \tau_{L}^{2}\right)$, on which the intensity increases with time. Perturbations propagate along the characteristics with speed of sound in water and the gold of layer heating. The laser energy is absorbed in the skin layer of the metal near the gold-water contact boundary. Acoustic disturbances are created due to temperature growth and thermal expansion of the substance in the heated layer with the current thickness of the order of $d_{T}$.

The contact boundary reaches the melting temperature $T_{\mathrm{m}}$ at the time moment $t \approx$ $-0.6 \mathrm{~ns}$, see figure 9 and pictures 10,11; to better understand the melting, the phase diagram of gold is shown in the figures 10, 11. Formation of a tooth on a pressure profile in figure 9 associated with the appearance of the liquid phase and with the difference scheme used. Outside the tooth, the pressure profile remains smooth, see figure 12. A similar detail with profile strengthening due to the beginning of melting was observed earlier in the works [43] (see figure 6 and 7 in this article), [44] (see figure 2 and 3 in this article; melting imprint in the profile of a simple Riemann wave profile), [45]. All these articles are devoted to ultrashort laser pulses when heating occurs isochorically. Then the melting section between solidus and liquidus is imprinted in the profiles of temperature and pressure. Then this spot begins to travel according to acoustic characteristics.

The work [11] also observed the strengthening of the Riemann simple wave profile, which (strengthening) is emitted from the melting front when the melting zone is switched from supersonic to subsonic propagation. If the works [43-45] are based on the hydrody- 


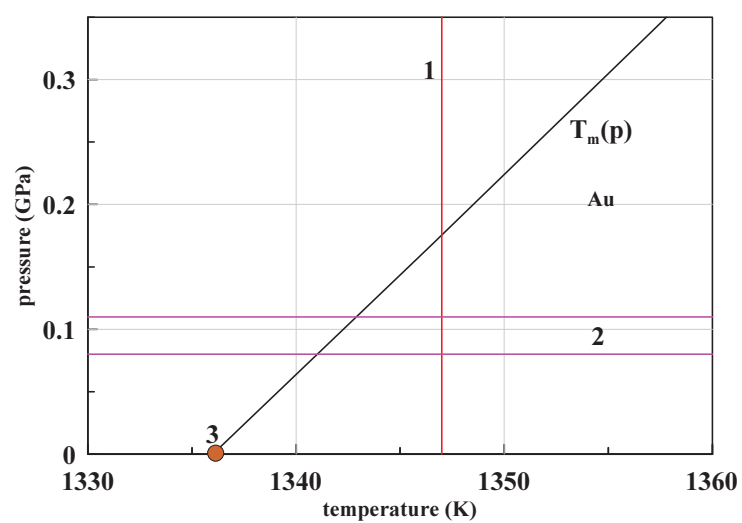

Fig.10 A small segment of the melting curve on the plane $T, p$ is presented. This is the segment near the triple point marked with marker 3. A more complete phase diagram in the pressure range of interest is shown in the following figure 11. At the triple point, the melting point of gold $(\mathrm{Au})$ is $1337 \mathrm{~K}$. The amplitude of the tooth pressure, taken from figure 9 , shown by lines 2 . Contact temperature $T_{\mathrm{cb}}=1347 \mathrm{~K}$ in figure 9 marked vertical line 1 . This temperature corresponds to the time $t=-0.6 \mathrm{~ns}$. As you can see, the temperature $T_{\mathrm{cb}}=1347 \mathrm{~K}$ is 5 degrees higher than the melting point $T_{\mathrm{m}}(p=943 \mathrm{bar})=$ $1342 \mathrm{~K}$. The pressure of 943 bar corresponds to the average pressure between horizontal lines 2 .

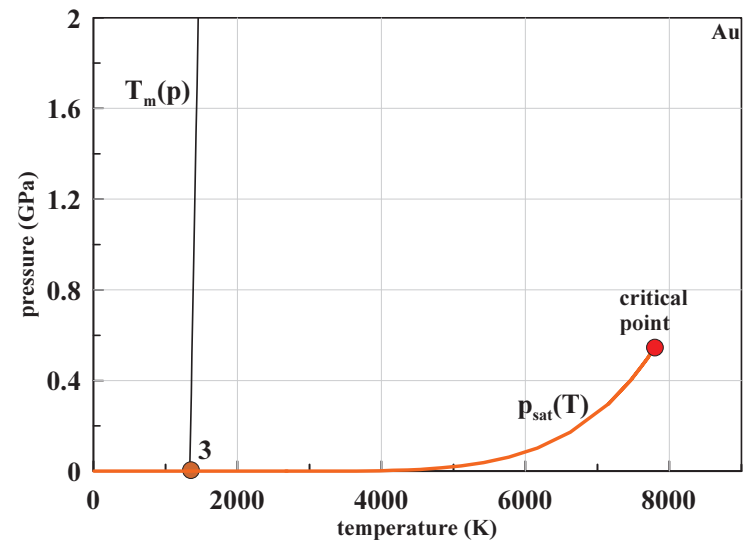

Fig.11 The figure shows the melting curve $T_{m}(p)$ or $p_{m}(T)$ and the dependence of the saturated vapor pressure on the temperature $p_{\text {sat }}(T)$. The data are taken according to the equation of state developed in the [46-51]. Marker 3 marked a triple point. The dependence $p_{\text {sat }}(T)$ ends at the critical point with the parameters $7800 \mathrm{~K}, 5300$ bar and $5.3 \mathrm{~g} / \mathrm{cm}^{3}[46-51]$. The vapor pressure at the triple point is negligible $p_{\text {sat }}(T=1337 \mathrm{~K})=$ $4.3 \times 10^{-8}$ bar; the concentration of gold atoms in a vapor is $2.3 \times 10^{11} \mathrm{~cm}^{-3}$. The boiling point is $p_{\text {sat }}(T=3243 \mathrm{~K})=1$ bar. 


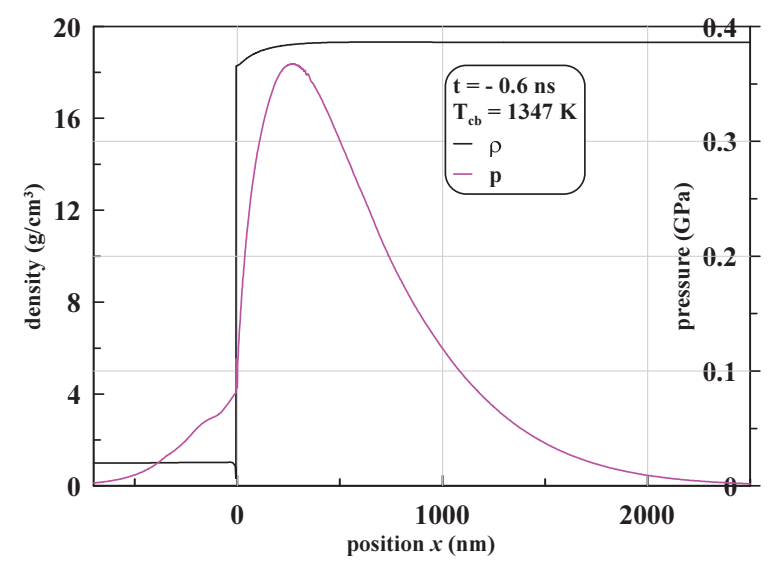

Fig.12 The density and pressure profiles at the same time point as shown in figure 9 . Acoustic perturbations (simple Riemann waves running into the water and into gold) outside the wave of pressure are smooth functions of the coordinate. Tooth in figure 9 on the scale of $x$, adopted in this figure, is a very narrow splash at $x \approx 0$.

namic code, then [11] is used molecular dynamics (MD). In MD, melting proceeds as in nature. The MD automatically takes into account the possible real effects of the melting nonequilibrium; in hydrodynamic modeling there is no disequilibrium, an equilibrium equation of state is used. In the MD pressure profiles, the melting site is smoothed, but remains noticeable. And if in [43-45] the plastic equation of state of matter in the solid phase is used (there are no elastic shock waves) [46-51], then MD [2,5,7-9, 11, 23, 52,53] clearly shows the elastic-plastic transition; a detailed experimental and theoretical studies of elastic-plastic transition in ultrashort loading were carried out in the works of Ashitkov, Kanel, Agranat, et al. [6,54,55], see also [56,57]. It turns out [11], that there is a superposition of two effects (A) of the ablation imprint of melting on the characteristics and (B) of the formation of an elastic shock wave. The strengthening of the acoustic profile section by melting accelerates the formation of an elastic shock wave - the simple Riemann wave is tilted earlier because of the presence of a steeper section.

Figure 13 shows the density, temperature, and pressure profiles at time $t=-0.2 \mathrm{~ns}$. At this point, the gold absorbed $29 \%$ of the laser pulse energy, i.e. $29 \%$ of $0.9 \mathrm{~J} / \mathrm{cm} 2$. The thickness of the gold melt layer in figure 13 is $0.25 \mu \mathrm{m}$. At stage II, shown in figure 13, the laser continues actively heat gold in the heating layer $d_{T}$. This heating increases and raises the pressure in the layer $d_{T}$. Therefore, the pressure profile has the form of a bump with a maximum slightly to the right of the heating layer $d_{T}$. On the right and left acoustic wings a trace from the beginning of melting is clearly visible. The specified trace (or imprint), as discussed above for ultrashort pulses, has the form of a section with a steeper pressure stroke.

Oscillations running to the right and to the left of the melting front are associated with the error of the difference scheme. Oscillations propagate along the characteristics. The calculation uses a one-dimensional hydrodynamic code with division into steps (cells) at the Lagrangian coordinate $x^{0}$, see $[35,36,38-42]$. The partition pitch (the length of the Lagrangian cell) is $\Delta x^{0}$ uniform over the integration interval. In the described calculation, 


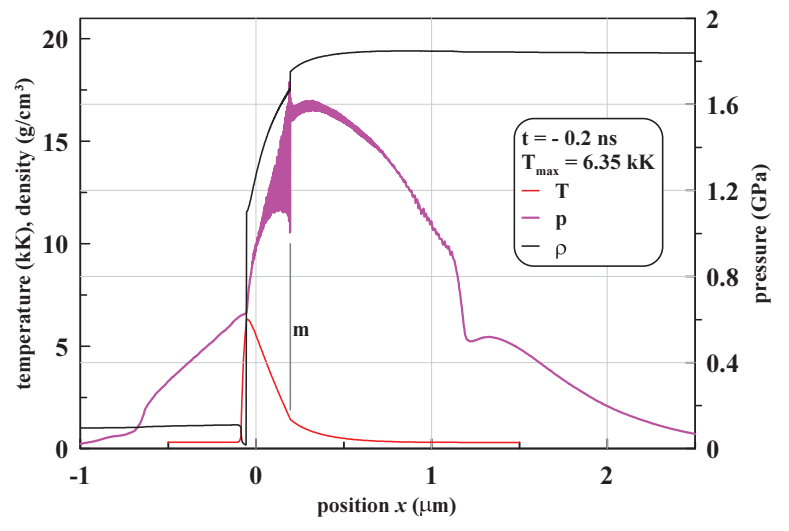

Fig.13 The middle of stage II (melting stage). The melting front is marked by the vertical $\mathrm{m}$. The strong heating of gold with a laser continues, the contact temperature is 6.35 $\mathrm{kK}$, the melt thickness is a quarter of a micron. Because of the heating, the pressure increases with time and the pressure profile looks like a mound with a maximum slightly to the right of the heating layer, cf. from figure 7 . Two steep sections with high pressure gradients are clearly visible on the pressure profile. They are at $x=-0.6 \mathrm{~m}$ and $x=1.2$ $\mathrm{m}$. Behind these areas, the $p$ profile smoothly falls outward from the contact. The outer portions are acoustic waves emitted from the heating layer in stage I (i.e. before melting). Tilting of simple Riemann waves on steep sections due to the focusing characteristics leads to the nucleation of shock waves on these sections. Focusing is due to non-linear effects in simple waves. Rollover occurs later in time with respect to the moment of time $t=-0.2 \mathrm{~ns}$, shown in this figure.

$\Delta x^{0}=1 \mathrm{~nm}$ is assumed. The time step is $10 \mathrm{fs}$. The velocity of the melting front on matter is $u_{\mathrm{m}}=400 \mathrm{~m} / \mathrm{s}$ in the considered time interval. This means that the melting front one step (one cell) along the Lagrangian grid passes over a time interval $\Delta t_{\mathrm{m}}=\Delta x^{0} / u_{\mathrm{m}}=2.5$ ps. At this moment, the state of matter in the cell $\Delta x^{0}$ changes abruptly from the state of the solid phase to the state of the liquid phase.

One more time. Consider a cell that is adjacent to the melting front but not yet melted. It remains in a solid state 250 steps in time. And then literally in one step in time passes into the liquid phase. This transition creates an oscillation due to the density difference in the solid and liquid phases; as the cell expands, passing into the liquid. Further, this oscillation is carried by the characteristics departing from the melting front to the right and to the left. The spatial length of the oscillation is $\Delta t_{\mathrm{m}} c_{s} \approx 5 \mathrm{~nm}$ at the speed of sound in gold at a melting temperature of about $2 \mathrm{~km} / \mathrm{s}$.

We note that in the case of a relatively long (subsonic) laser pulse considered here with $\tau_{L}=0.5 \mathrm{~ns}$, the melting front is just the front. Its thickness is equal to the width of a single Lagrangian cell $\Delta x^{0}$. If we reduce the pitch $\Delta x^{0}$, then the oscillation length will be proportionally reduced; of course, the step can be greatly reduced so that the melting front is blurred into a transition zone with varying phase composition. The sharpness of the transition between the phases means that the melting process is described quite well in the approximation of the Stefan problem. In this sense, the subsonic laser pulse is qualitatively different from the "supersonic" pulse. The latter corresponds to the 
ultrashort pulse. The impulse becomes supersonic when $\tau_{L}<d_{T} / c_{s}$. In the opposite case, the pulse was named above slow or subsonic. In the supersonic case, there is a transition zone of sufficiently Lagrangian cells of mixed composition, in which the solid and liquid phases coexist [43-45]; therefore, in the case of ultrashort exposure, there are no such pronounced oscillations as in figure 13. The composition gradually changes from pure solid to pure liquid at the edges of the transition zone.

The presence of oscillations is associated with the difference character of approximation. As one moves away from the melting front, the oscillation amplitude decreases. The first oscillation, which was formed at the time of the beginning of melting (shown in the figures 9, 10 and 12), remains pronounced at subsequent points in time, see, for example, fig 13. In figure 13 steep sections on the pressure profile correspond to this oscillation. There are two such sites. At the moment of $t=-0.2 \mathrm{ps}$, one of them is in water at $x \approx-0.6 \mu \mathrm{m}$, while the second is in gold at $x \approx 1.2 \mu \mathrm{m}$, see figure 13 . The speed of sound in gold is greater, so the area in gold is located farther from the contact. The subsequent several oscillations, which were emitted by the melting front after the first, have already attenuated substantially. Their amplitude is small compared with recently emitted oscillations.

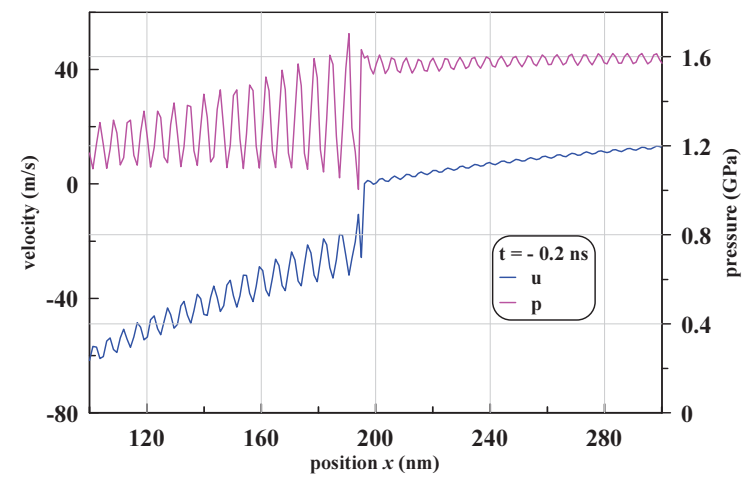

Fig.14 The jump associated with the melting front separates the liquid phase on the left and the solid phase on the right. The relation between the jump amplitudes and the direct Rayleigh is discussed in the text. The movement of the melting front is subsonic, the Mach number is about 0.17 .

Due to the expansion of the metal during melting, a velocity jump occurs at the melting front. The amplitude of the jump is approximately $\Delta u_{\mathrm{m}} \approx 20 \mathrm{~m} / \mathrm{s}$. With the acceleration of the substance at the intersection of the melting front, the existence of a small pressure jump at the front is connected. Speed and pressure jumps are shown in figure 14. The pressure jump amplitude is $\Delta p_{\mathrm{m}} \approx 0.18 \mathrm{GPa}$. The velocity of the melting front relative to the substance is about $u_{\mathrm{m}} \approx 400 \mathrm{~m} / \mathrm{s}$. At the melting front, conservation laws are fulfilled. The Rayleigh line $\Delta p_{\mathrm{m}}=j \Delta u_{\mathrm{m}}$ follows from the conservation of mass and momentum; here $j=\rho u, u$ is the velocity of matter relative to the front, i.e. $u_{\mathrm{m}}$. The values of $\Delta p_{\mathrm{m}}, \Delta u_{\mathrm{m}}$ and $u_{\mathrm{m}}$ from our calculation satisfy the relation that is the direct Rayleigh.

Figure 15 shows the portion of the temperature profile near the melting front. On the profile, points mark the division points into Lagrangian cells. Direct $1426 \mathrm{~K}$ gives the 


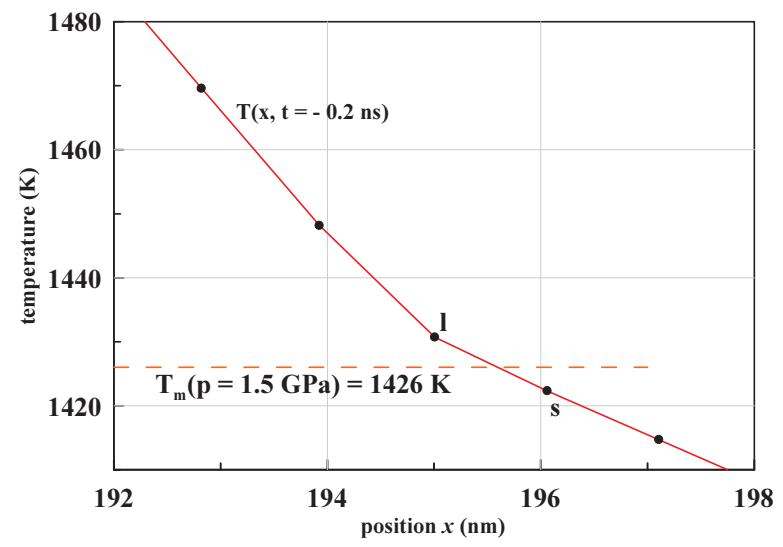

Fig.15 Temperature distribution over Lagrangian nodes/cells near the melting front at time-0.2 ns. Explanations regarding the jerks of state from solid to liquid and relative to the temperature gradient are given in the text. The state changes when the node s (solid phase) reaches a melting point of $1426 \mathrm{~K}$. This is the melting point at a pressure of 1.5 $\mathrm{GPa}$ in the vicinity of the melting front, see figure 14.

melting point according to the melting curve $T_{\mathrm{m}}(p)$. This curve is shown in the figures 10 and 11. The melting point of $1426 \mathrm{~K}$ is reached at a pressure of $1.5 \mathrm{GPa}$. The pressure value of $1.5 \mathrm{GPa}$ in the region of the melting front at the time $t=-0.2 \mathrm{~ns}$ is taken from figure 14 .

The temperature difference between points s (solid phase) and l (liquid phase) in figure 15 is equal to $8 \mathrm{~K}$. The temperature in the Lagrangian cell s grows at a rate of $0.03 \mathrm{~K}$ in one integration step over time. Over the order of 100 time steps, the temperature in the Lagrangian cell s will exceed the melting point of $1426 \mathrm{~K}$. At the same time, the cell will change its status - it will turn from solid to liquid (melt). The formation of the next oscillation is due to such melting jerks.

The temperature gradient is higher in the liquid phase, see figure 13 and 15 . Two factors determine this effect. First, melting occurs at this stage. I. e., new and new portions of solid gold are melted. In order to cover the costs associated with the latent melting heat $Q$, it is necessary that the heat flux $q_{1}$ to the front exceed the heat flux $q_{\mathrm{s}}$ from the front: $q_{1}-q_{\mathrm{s}}=Q \rho_{\mathrm{s}} u_{\mathrm{m}}$, where $Q$ - the heat of fusion per unit mass. Secondly, the coefficient of thermal conductivity is about 2-2.5 times lower in the liquid phase; the thermal conductivity model used in the calculation takes into account the change in thermal conductivity during melting. Therefore, even at $Q=0$, the temperature gradient will be higher in the liquid.

In the case of long pulses, the resulting pressure is small (compared with the bulk modulus of the metal). Therefore, the substance is located along the boiling curve in accordance with the temperature distribution in gold, see figure 16; the boiling curve is called the right branch of the binodal (phase coexistence curve). I.e., the extension of matter is mostly due to the heat. The available pressure has little effect on the density (in the sense of deviation from the boiling curve).

In figure 17 the hydrodynamic profile is compared with the boundaries of the phase transitions of the first kind on the plane $\rho, p$. We see that on shown in figure 17 the 


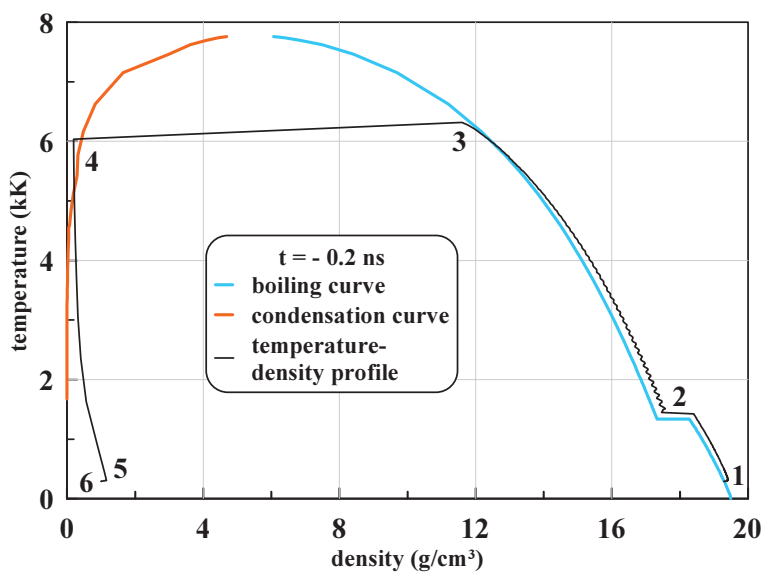

Fig.16 The state of matter in the phase diagram at time point of- 0.2 ns. Together with the phase equilibrium curves, the density and temperature profiles are constructed in parametric form: $\rho(x, t=-0.2 \mathrm{~ns}), T(x, t=-0.2 \mathrm{~ns})$. The parameter that runs through the profile is the coordinate $x$. Points 1-6 refer to: 1 -undisturbed gold deep in the target, 2-melting front repeating the density jump at the triple point (figure 13-15), 3-maximum temperature on contact with water (figure 13), 3-4-density jump from gold to water (figure 13), 4-hot water adjacent to the contact, 4-5 temperature profile in water, 6-water far from the contact. Of course, the states of water are irrelevant to the gold condensation curve. Under the influence of the pulse, the temperature of gold rises. Therefore, point 3 rises higher and higher along the boiling curve. However, there is no gold evaporation, since the pressure is higher than the saturated vapor pressure, see figure 11. Evaporation will start when point 3 reaches the critical point. This issue is devoted to the next section of work. 


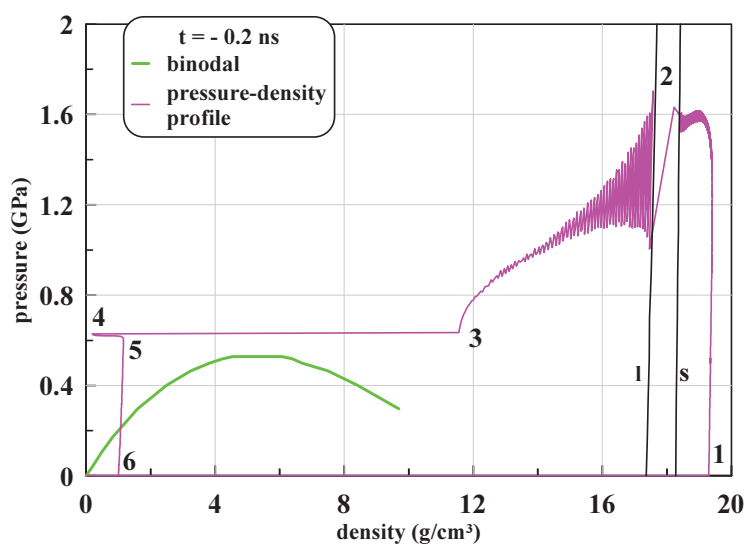

Fig.17 Here, the hydrodynamic profile is built together with the phase equilibrium curves on the $\rho, p$. plane. The numbers $1-6$ indicate the same states as in the previous figure 16 . We see that the pressure at the hottest point of gold is still above the critical pressure of gold. The hottest is point 3. It is on contact with water. The letters s (solidus) and 1 (liquidus) indicate melting curves bordering the corridor of the existence of a mixture of solid and liquid phases. The intersection of this corridor with the instantaneous profile of the density and pressure distributions corresponds to segment 2 in figure 16. The pressure at the intersection of the melting front abruptly decreases, cf. with pictures 13 and 14

moment of time all gold (points 1-2-3) is still located to the right of the critical density of gold $5.3 \mathrm{~g} / \mathrm{cm}^{3}$. Additional heating is needed to exceed the critical temperature (see figure 16) and lower the density below the critical value (see figure 17).

\section{The excess of the critical temperature of gold, the appearance of a contact layer of gaseous gold}

Over time, the temperature of the metal near the contact rises under the action of the laser. As mentioned above, the flow in its development goes through several stages: stage I ends with the start of melting; stage II is completed with the achievement of the critical temperature of the metal. At stage III, three layers are adjacent to the metal contact with water. With the transition from stage II to stage III begins the formation of a layer of gold in the gaseous state. So at stage III, a layer of gaseous gold is located near the contact, then a layer of melt follows, and then there is a solid phase.

Figures 18-21 show the situation at time $t=0$. Stage III begins: the contact temperature exceeds the critical temperature $T_{\mathrm{cr}}=7.8 \mathrm{kK}$, and the density falls below the critical value $\rho_{\mathrm{cr}}=5.3 \mathrm{~g} / \mathrm{cm}^{3}$. But the pressure is about 4 times the critical value of $p_{\text {cr }}=0.53$ GPa. As is known, the density range $\rho<\rho_{\text {cr }}$ is related to the gas phase. At a density of $\rho>\rho_{\text {cr }}$, the substance is assumed to be in the liquid phase. Of course, above the critical point such a division is conditional. A substance at $\rho \sim \rho_{\text {cr }}$ is called a fluid or nonideal gas. We are talking about the gaseous state, not the gas state, in the case of nonideal gas. Although in the case shown in figure 18-21, the gold pressure in the gas layer between the boundaries 2 and 3 in figure 19 is close to the pressure given by the formula for the 


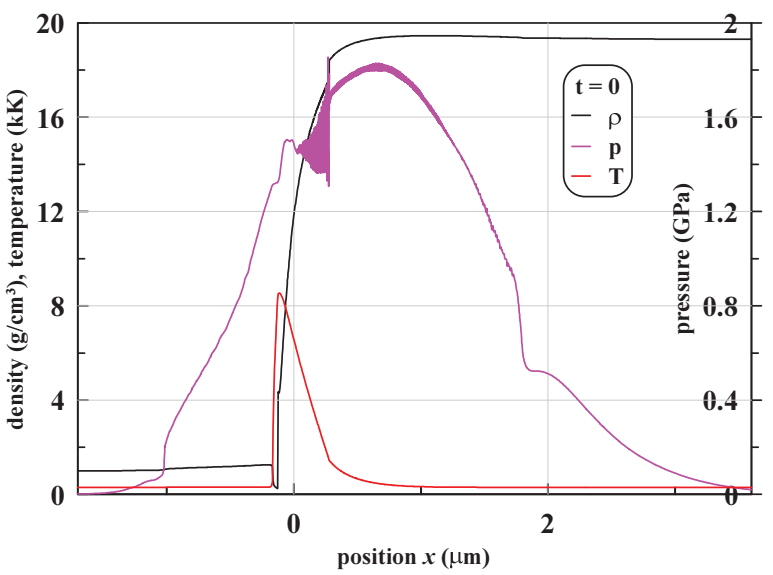

Fig.18 Profiles of density, temperature and pressure at time $t=0$. The pressure profile contains information about changes in the intensity of the laser pulse over time. The moment $t=0$ is close to the moments of overturning of simple waves in water and gold: the steep sections on the pressure profile are close to the vertical segments at the points of inflection. By the time $t=0$, gold absorbed half the energy $F_{\text {abs }} / 2$ of the incident pulse $I(t)=I_{0} \exp \left(-t^{2} / \tau_{L}^{2}\right)$. In the calculation described in this paper, we have: $\tau_{L}=0.5$ ns, $F_{\text {abs }}=900 \mathrm{~J} / \mathrm{cm}^{2}$. At $t>0$, the intensity of $I(t)$ decreases as compared with the maximum value of $I_{0}$.

ideal gas $p=n k_{B} T$ for the density and temperature in this layer. Note that according to used in the calculation of the equation of state of gold [46-51], compressibility factor of $p /\left(n k_{B} T\right)$ is equal to 0.3 at the critical point.

By the time point $t=0$ gold absorbed half the energy $F_{\text {abs }}$ of the pulse. From the figures 18-21 it follows that the gas layer of gold 2-3 (see figure 19) is formed at the moment $t \approx 0$. The coincidence with the maximum of the pulse $\tau_{L}=0.5 \mathrm{~ns}, F_{\mathrm{abs}}=900$ $\mathrm{J} / \mathrm{cm}^{2}$ occasional. Indeed, if the energy of $F_{\text {abs }}$ were greater, then the achievement of the gas state would occur before the maximum pulse $I \propto \exp \left(-t^{2} / \tau_{L}^{2}\right)$.

In figure 20 layers are shown: 1-2 hot water, coordinates $x=-167$ and $-125 \mathrm{~nm}$, thickness $42 \mathrm{~nm}$; a layer of gas gold 2-3 with coordinates -125 and $-113 \mathrm{~nm}$, thickness 12 $\mathrm{nm}$ and a layer of melt almost $400 \mathrm{~nm}$ thick. The melting front coordinate at time $t=0$ is $278 \mathrm{~nm}$. The coordinates of $x$ are measured from the initial position of the contact boundary.

We emphasize that in the gas state layer 2-3 in figure 19 falls around the critical point from above. That is, the layer $2-3$ is not formed as a result of evaporation of the heated condensed matter. Pressure in the region 1-2-3 in figure 19 above the saturated vapor pressure $p_{\text {sat }}(T)$. This pressure is above the maximum value that the function $p_{\text {sat }}(T)$ can have, i.e. above the critical pressure $p_{\mathrm{cr}}$, see figures 11 and 21 . Layer $2-3$ in figure 19 is formed as a result of continuous temperature growth on the temperature profile $T(x, t)$ of gold over time $t$ during the action of the laser pulse: $T\left(x^{0}, t_{2}\right)>T\left(x^{0}, t_{1}\right)$, if $t_{2}>t_{1}$, here $x^{0}$-Lagrangian coordinate $O n$ the phase plane $\rho, T$ this profile is located along the melting curve, see figures 16 and 20. To the instantaneous profile $T(x, t), \rho(x, t)$ there correspond points: 1 (state far in volume)-2 (melting front)-3 (contact) in the figures 16 


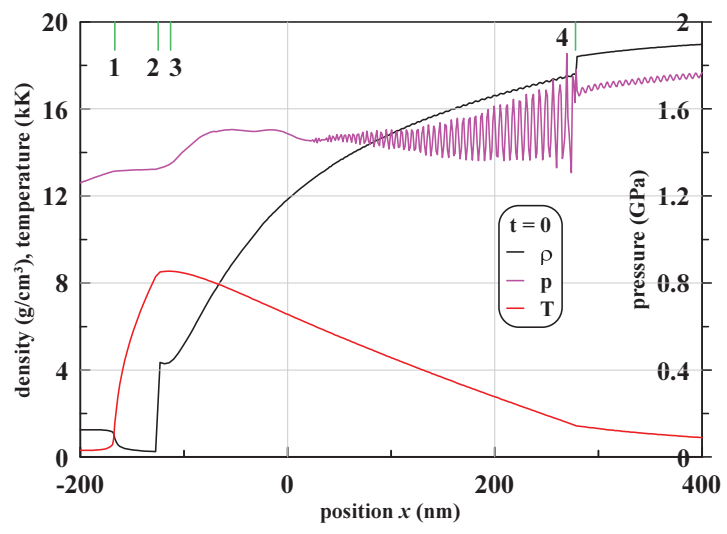

Fig.19 The structure of the contact zone at $t=0$ is shown. Strips 1, 2, 3 and 4 mark the boundaries of the layers. To the left of 1 is cold dense water, the thermal conductivity of water is calculated from the data taken from the literature. Between the boundaries 1 and 2 there is a layer of hot partially dissociated water, 2 - gold-water contact. The equation of state of water is taken from [58]. Layer 2-3 is gaseous gold. Layer 3-4 occupies the liquid phase, 4 - the melting front, it moves relative to the substance to the right. To the right of 4 is the solid phase.

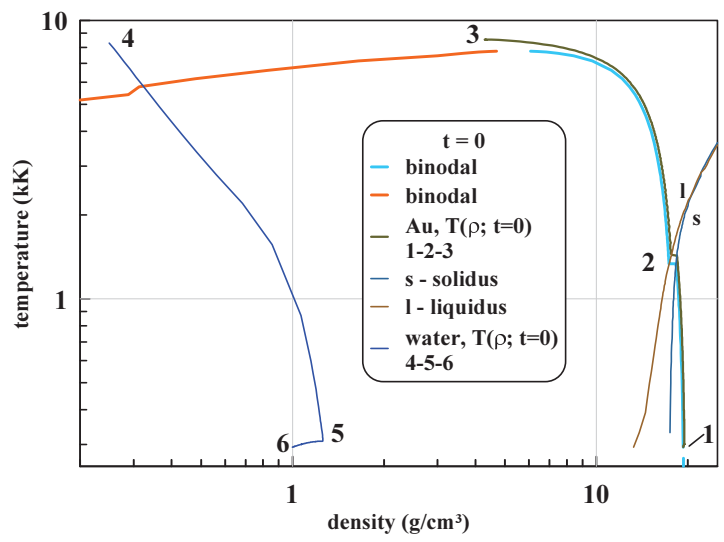

Fig.20 Temperature profile $T(\rho ; t=0)$ on the phase plane $\rho, T$. The gradual heating by the laser leads to the fact that the temperature on the profile $T(\rho ; t=0)$ rises above the critical value $T_{\mathrm{cr}}=7.8 \mathrm{kK}$. The single (for water and $\mathrm{Au}$ ) profile $T(\rho ; t=0)$ is divided into two 1-2-3 (Au) and 4-5-6 (water) pieces. A density jump of 3-4 refers to the contact boundary, cf. from figure 16, where a single profile is shown. Au and water densities at the contact are 4.35 and $0.25 \mathrm{~g} / \mathrm{cm}^{3}$. Curves s (solidus) and $\mathrm{l}$ (liquidus) are continued through the triple point to the metastable area [46-51]. Point 1 corresponds to gold at a great distance from the contact, where the temperature is equal to room emperature 300 K. 


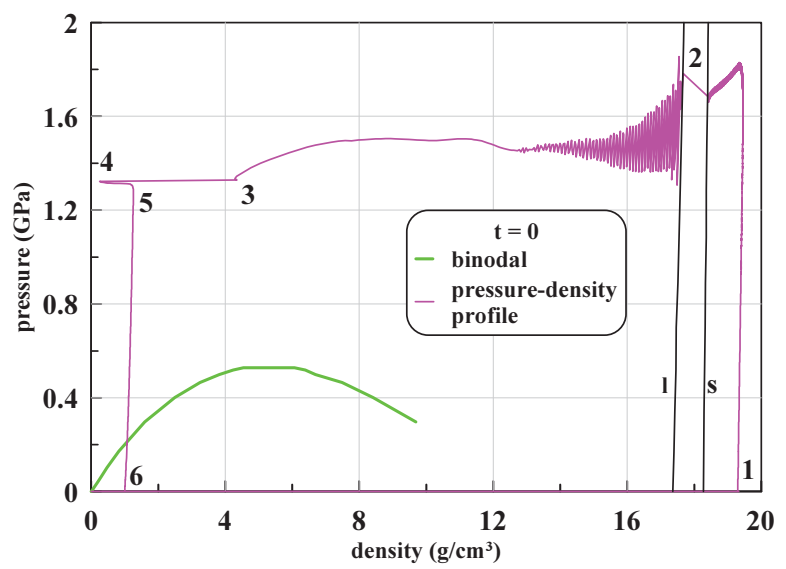

Fig.21 Pressure profile $p(\rho ; t=0)$ on the phase plane $\rho, p$. For the time elapsed from the previous time point $t=-0.2 \mathrm{~ns}$, see figure 17 , the pressure has increased significantly. The maximum pressure is in the solid phase behind the melting front. The meaning of numbers $1-6$ is the same as in figures 16,17 and 20 . Note that the $1-2-3$ profile, which was just above the boiling curve in figure 20 and 16, here it is far removed from the boiling curve. This is due to the low compressibility of the condensed phase.

and 20 .

Due to the increase in temperature at the contact, the top point 3 of the profile $T(x, t), \rho(x, t)$ moves up along the melting curve, cf. figures 20 and 16 . Point 3 in the figures 20 and 16 reaches the critical value $T_{\text {cr }}=7.8 \mathrm{kK}$ and then (with continued heating) it begins to exceed this value if the energy input $F_{\text {abs }}$ is large enough. The temperature profile $T(\rho ; t)$ in figure 20 goes slightly above the boiling curve, because the pressure is higher than $p_{\text {sat }}(T)$.

Let the saturated vapor above the liquid phase form as a result of the evaporation of this liquid. Then there are two states on the phase plane $\rho, T$ : one on the boiling curve and the other on the condensation curve. The pressure in the vapor-liquid system is equal to the saturated vapor pressure $p_{\text {sat }}(T)$, see figure 11 . In our situation, as it is said, evaporative formation of a layer of pure gold vapor (without water) is impossible until the condition $p>p_{\text {sat }}(T)$ is satisfied.

Compare the number of gold atoms that have passed the gas-vapor phase by evaporation (path B) and by heating above the critical temperature point $T_{\mathrm{cr}}=7.8 \mathrm{kK}$ by $\Delta T$ of the order of one or several thousand degrees (path A). The evaporation of gold atoms into water takes place even in the profile situation shown in figure 16. The corresponding number of evaporated atoms $N_{B}$ is small compared to the number of atoms $N_{A}$ in interlayer $2-3$ in figure 19. In our code with the Lagrangian coordinate $x^{0}$ there are no mixed cells in which both gold atoms and water molecules would be present. Therefore, the processes of evaporation into water and mutual diffusion are not taken into account. An molecular dynamics approach [40] is required to analyze these processes.

As mentioned, the figures 18-21 show the situation at the time $t=0$ of the laser pulse maximum. Then the intensity absorbed in the metal begins to decrease with time. However, the growth of the temperature and pressure maxima $T_{\max }(t), p_{\max }(t)$ continues 
for some time, that is, these maxima are shifted in time relative to the intensity maximum $I(t)$. Stage IV starts when the formation of a dip in the pressure profile begins. The following section is devoted to this topic.

\section{Emission of acoustic disturbances from the heating layer}

Acoustic disturbance tracing the history of heating $I(t): I(t) \rightarrow T(x \sim 0, t) \rightarrow p(x \sim 0, t)$ $\rightarrow p(x \pm c t)$, where $x \sim 0$ is the area of heating, the letter $c$ with the plus sign is the wave going into the water. At the same time, $C$ is the speed of sound in water. Similarly, $c$ minus refers to the wave propagating in gold. Fig 22 shows the development of the acoustic field at the final stages of laser action, when the intensity of $I(t)$ decreases.

The increase in the maximum temperature of $T_{\max }(t)$ (over the instantaneous temperature field) continues at $t>0$, despite the decrease in the intensity of $I(t)$. Indeed, energy is still being supplied to the system. A slow decrease in the value of $T_{\max }(t)$ begins at $t \approx 0.4 \mathrm{~ns}$ when the thermal conductivity losses begin to exceed the laser heating power. The highest temperature in space and time $T_{\max -x t}$ is $11.3 \mathrm{kK}$ for the considered laser pulse. Radiation losses $\sigma_{\mathrm{SB}} T^{4} \sim 10^{9} \mathrm{~W} / \mathrm{m}^{2}$ in the considered range of times and temperatures are small in comparison with the electron heat flux in the metal $\kappa \mathrm{d} T / \mathrm{d} x \sim 10^{12}$ $\mathrm{W} / \mathrm{m}^{2}$ at $\kappa \sim 100 \mathrm{~W} / \mathrm{m} / \mathrm{K}, \mathrm{d} T \sim 8 \mathrm{kK}, d x \sim 300 \mathrm{~nm}$.

At $t \approx 0.3 \mathrm{NS}$ the formation of a dip on the pressure profile $p(x, t)$ in the area of the heating layer begins. This is shown in figure 22. The temperature rise in the heating region $\mathrm{d} T / \mathrm{d} t$ first slows down and then stops. Accordingly, the thermal expansion of gold stops. Namely, this expansion is associated with an increase in pressure in the heating region, which is emitted from this area by acoustic characteristics.

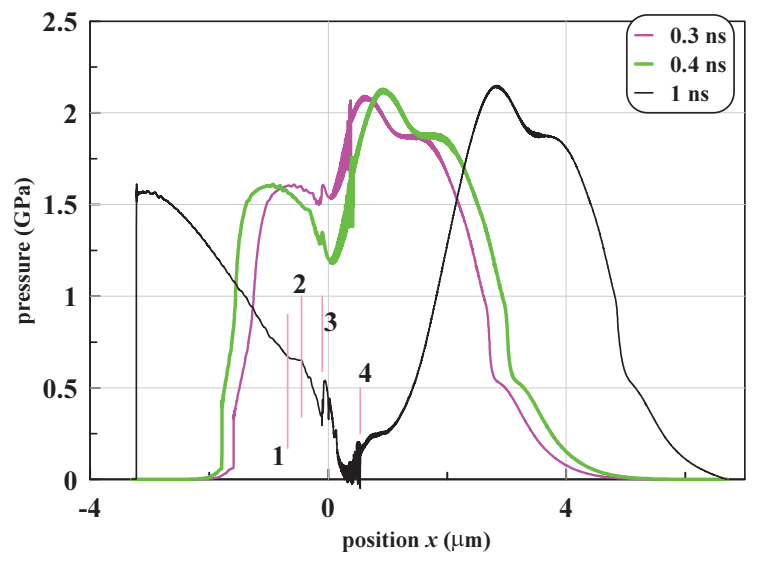

Fig.22 Evolution of the pressure field after the start of a decrease of the absorbed laser pulse intensity $I(t) \propto \exp \left(-t^{2} / \tau_{L}^{2}\right), t>0$. Recall that $\tau_{L}=0.5 \mathrm{~ns}$. At times about $t=0.3$ ns, the formation of a dip in the pressure profile begins due to a decrease in the rate of heating of the substance. At $t \approx 1$ ns there is a complete separation of waves running to the left (into the water) and to the right (into gold). Points 1, 2, 3 and 4 mark the boundaries of the layers. As in figure 19, these are the boundaries of the hot water layer $1-2$, the gaseous gold layer $2-3$ and the melt layer $3-4$ at the time $t=1 \mathrm{~ns}$. 
The beginning of stage IV is connected with the moment of time $t \approx 0.3$ ns with the formation of a dip in the $p$ profile. Gradually, a single pressure bump breaks up into two different waves extending into gold and water, see figure 22 . Herewith the pressure in the contact zone is reduced to a pressure that is determined by heated gold and hot water.

A shock wave is formed from a steep section in the water in figure 22; the existence of two steep sections was discussed in figures 13 and 18; this is the transition from stage I to stage II. The volumetric water module of $2.25 \mathrm{GPa}$ is almost two orders of magnitude lower than the gold module of $180 \mathrm{GPa}$. Therefore, the tilting of a steep section in water occurs much earlier than in gold. Although the water pressure is slightly lower than gold, see figure 22. In the shock wave, the heating history of $I(t)$ gradually disappears, reflected in the $p(x \pm c t)$ profile of the Riemann simple wave. Gradually, the wave transforms into a triangular shock wave, cf. the profiles at time points of 0.3 and 0.4 ns with the profile at time of $1 \mathrm{~ns}$ in figure 22 .

The evolution of the shock wave in the metal is important for laser peening. For peening, it is necessary that (i) a shock wave be formed in the metal and (ii) that the amplitude of this wave be above the elastic limit. Then an elastic plastic wave propagates into the metal. Plastic deformations remaining behind the front of a plastic wave are due to the hardening of the surface layer of the product with respect to fatigue failure and corrosion. The solution of the problem of elastic-plastic wave is beyond the scope of this presentation. The complexity here is in the end-to-end consideration of the elastic-plastic behavior together with the phenomena of phase transitions of the first kind: melting/crystallization and condensation. Previously, this problem was solved for the case of ultrashort pulse: see work with molecular dynamics [7-9,11], as well as hydrodynamic studies in which multiphase and phase transitions are embedded together with elasto-plasticity $[52,59,60]$.

In connection with the applications associated with the formation of colloids of nanoparticles and laser peening, the earlier stages of the action of a nanosecond pulse on a gold target in water have been poorly studied. Thermophysical processes of formation and emission of simple Riemann waves from the heating region are considered. The history of the contact zone is investigated: gradual heating of gold and water: the formation of a hot water layer and a hot gold layer.

Acknowledgement. Authors (INA and VVZ) acknowledge support from the Russian Science Foundation, grant No. 19-19-00697.

\section{References}

[1] S.I. Anisimov, N.A. Inogamov, Singular self-similar superdense compression regimes for laser targets, J. Appl. Mech. Tech. Phys. 21, 449 (1980)

[2] V.V. Zhakhovsky et al., MD simulation of steady shock-wave fronts with phase transition in single-crystal iron, AIP Conf. Proc. 1793, 070003 (2017)

[3] V.V. Zhakhovskii et al., Shock wave structure in Lennard-Jones crystal via molecular dynamics, Phys. Rev. Lett. 83 (6), 1175 (1999)

[4] S.I. Anisimov, Yu.V. Medvedev, Interaction of collisionless shock waves in a plasma, Sov. Phys. Tech. Phys. 33 (10), 1123-1129 (1988)

[5] M.B. Agranat et al., Strength properties of an aluminum melt at extremely high tension rates under the action of femtosecond laser pulses, JETP Lett. 91 (9), 471 (2010)

[6] Ashitkov et al., Behavior of aluminum near an ultimate theoretical strength in experiments with femtosecond laser pulses, JETP Lett. 92 (8), 516 (2010) 
[7] V.V. Zhakhovskii, N.A. Inogamov, Elastic-plastic phenomena in ultrashort shock waves, JETP Lett. 92 (8), 521 (2010)

[8] V.V. Zhakhovsky et al., Two-Zone Elastic-Plastic Single Shock Waves in Solids, Phys. Rev. Lett. 107, 135502 (2011)

[9] N.A. Inogamov et al., Superelasticity and the propagation of shock waves in crystals, JETP Lett. 93 (4), 226 (2011)

[10] S.I. Ashitkov et al., Achievement of ultimate values of the bulk and shear strengths of iron irradiated by femtosecond laser pulses, JETP Lett. 98 (7), 384 (2013)

[11] B.J. Demaske et al., Ultrashort shock waves in nickel induced by femtosecond laser pulses, Phys. Rev. B 87, 054109 (2013)

[12] S.I. Ashitkov et al., Mechanical and optical properties of vanadium under shock picosecond loads, JETP Lett. 101 (4), 276 (2015)

[13] S.I. Ashitkov et al., Strength of liquid tin at extremely high strain rates under a femtosecond laser action, JETP Lett. 103 (8), 544 (2016)

[14] V.V. Zhakhovsky et al., Super-elastic response of metals to laser-induced shock waves, AIP Conf. Proc. 1464, 102 (2012)

[15] V.V. Zhakhovsky et al., Single two-zone elastic-plastic shock waves in solids, AIP Conference Proceedings 1426, 1227 (2012)

[16] B.J. Demaske et al., Ablation and spallation of gold films irradiated by ultrashort laser pulses, Phys. Rev. B 82, 064113 (2010)

[17] B. Albertazzi et al., Dynamic fracture of tantalum under extreme tensile stress, Science Advances 3 (6) e1602705 (2017)

[18] S.I. Anisimov et al., Application of high-power lasers to study matter at ultrahigh pressures, Sov. Phys. Usp. 27 (3), 181 (1984)

[19] E.B. Zaretsky, G.I. Kanel, Effect of temperature, strain, and strain rate on the flow stress of aluminum under shock-wave compression, Journal of Applied Physics 112 (7), 073504 (2012)

[20] G.I. Kanel, Rate and temperature effects on the flow stress and tensile strength of metals, AIP Conference Proceedings 1426, 939 (2012)

[21] E.B. Zaretsky, G.I. Kanel, Plastic flow in shock-loaded silver at strain rates from 104 $s-1$ to $10^{7} \mathrm{~s}-1$ and temperatures from $296 \mathrm{~K}$ to $1233 \mathrm{~K}$, Journal of Applied Physics 115 (24), 243502 (2014)

[22] V.V. Zhakhovsky et al., Elastic-plastic collapse of super-elastic shock waves in facecentered-cubic solids, Journal of Physics: Conference Series 500, 172007 (2014)

[23] R. Perriot et al., Evolution of elastic precursor and plastic shock wave in copper via molecular dynamics simulations, Journal of Physics: Conference Series 500, 172008 (2014)

[24] K. Sokolowski-Tinten et al., Transient states of matter during short pulse laser ablation, Phys. Rev. Lett. 81 (1), 224 (1998)

[25] N.A. Inogamov et al., Expansion of matter heated by an ultrashort laser pulse, JETP Lett. 69, 310 (1999)

[26] V.V. Zhakhovskii et al., New mechanism of the formation of the nanorelief on a surface irradiated by a femtosecond laser pulse, JETP Lett. 87 (8), 423 (2008)

[27] S.I. Ashitkov et al., Formation of Nanocavities in Surface Layer of Aluminum Target irradiated by Femtosecond Laser Pulse, JETP Letters 95 (4), 176 (2012)

[28] N.A. Inogamov et al., Surface nanodeformations caused by ultrashort laser pulse, Engineering Failure Analysis 47, 328 (2015)

[29] D. Karthik, S. Swaroop Laser peening without coating-an advanced surface treatment: A review, Mater. Manuf. Processes 32 (14), 1565 (2017) 
[30] C. Correa et al., Random-type scanning patterns in laser shock peening without absorbing coating in 2024-T351 Al alloy: A solution to reduce residual stress anisotropy, Opt. Laser Technol. 73, 179 (2015)

[31] J. Lam et al., Dynamical study of bubble expansion following laser ablation in liquids, Appl. Phys. Lett. 108 (7), 074104 (2016)

[32] S.A. Dyachkov et al., Explicit failure model for boron carbide ceramics under shock loading, J. Appl. Phys. 124 (8), 085902 (2018)

[33] J. Sanz et al., Quasi-steady expansion of plasma ablated from laser-irradiated pellets, Phys. Fluids 24 (11), 2098 (1981)

[34] J. Sanz, Self-consistent Analytical Model of the Rayleigh-Taylor Instability in Inertial Confinement Fusion, Phys. Rev. Lett. 73 (20), 2700 (1994)

[35] N.A. Inogamov et al., Jet Formation in Spallation of Metal Film from Substrate under Action of Femtosecond Laser Pulse, J. Exp. Theor. Phys. 120 (1), 15 (2015)

[36] N.A. Inogamov et al., Dynamics of Gold Ablation into Water, J. Exp. Theor. Phys. 127 (1), 79 (2018)

[37] R. Fabbro et al., Physical study of laser-produced plasma in confined geometry, J. Appl. Phys. 68 (2), 775 (1990)

[38] V.A. Khokhlov et al., Formation of solitary microstructure and ablation into transparent dielectric by a subnanosecond laser pulse, arXiv 1811.11990 cond-mat.mes-hall (2018)

[39] Y.V. Petrov et al., Laser-induced ablation of metal in liquid, arXiv 1812.09109 physics.comp-ph (2018)

[40] Y.V. Petrov et al., Condensation of laser produced gold plasma during expansion and cooling in water environment, arXiv 1812.09929 physics.comp-ph (2018)

[41] V.A. Khokhlov et al., Dynamics of supported ultrathin molybdenum films driven by strong short laser impact, J. Phys.: Conf. Ser. 1147 (1), 012066 (2018)

[42] N.A. Inogamov et al., Formation of a Single Microstructure and Ablation into a Transparent Dielectric Material under Subnanosecond Laser Irradiation, JETP Letters 108 (7), 439 (2018)

[43] N.A. Inogamov et al., Two-temperature relaxation and melting after absorption of femtosecond laser pulse, Appl. Surf. Sci. 255 (24), 9712 (2009)

[44] N.A. Inogamov et al., Acoustic probing of two-temperature relaxation initiated by action of ultrashort laser pulse, Appl. Phys. A 101 (1), 1 (2010)

[45] N.A. Inogamov et al., Laser Acoustic Probing of Two-Temperature Zone Created by Femtosecond Pulse, Contrib. Plasma Phys. 51 (4), 367 (2011)

[46] A.V. Bushman et al., Intense Dynamic Loading of Condensed Matter, (Taylor \& Francis Translation, London, 1993), $295 \mathrm{p}$

[47] K.V. Khishchenko et al., Metastable States of Liquid Tungsten Under Subsecond Wire Explosion, Intern. J. Thermophys. 23 (5), 1359 (2002)

[48] I.V. Lomonosov, Multi-phase equation of state for aluminum, Laser Part. Beams 25 (4), 567 (2007)

[49] rusbank, http://teos.ficp.ac.ru/rusbank/

[50] rusbank, http://www.ihed.ras.ru/rusbank/

[51] M.E. Povarnitsyn et al., A wide-range model for simulation of pump-probe experiments with metals, Appl. Surf. Sci. 258 (23), 9480 (2012)

[52] D.K. Ilnitsky et al., Two-temperature hydrodynamics of laser-generated ultrashort shock waves in elasto-plastic solids, J. Phys.: Conf. Ser. 500 (3), 032021 (2014)

[53] S.I. Ashitkov et al., The behavior of iron under ultrafast shock loading driven by a femtosecond laser, AIP Conf. Proc. 1793, 100035 (2017) 
[54] G.I. Kanel et al., Unusual plasticity and strength of metals at ultra-short load durations, Phys. Usp. 60 (5), 490 (2017)

[55] G.I. Kanel et al., Effect of Small Preliminary Deformation on the Evolution of Elastoplastic Waves of Shock Compression in Annealed VT1-0 Titanium, J. Exp. Theor. Phys. 127 (2), 337 (2018)

[56] V.H. Whitley et al., The elastic-plastic response of aluminum films to ultrafast lasergenerated shocks, J. Appl. Phys. 109 (1), 013505 (2011)

[57] J.C. Crowhurst et al., Invariance of the Dissipative Action at Ultrahigh Strain Rates Above the Strong Shock Threshold, Phys. Rev. Lett. 107 (14), 144302 (2011)

[58] R.I. Nigmatulin, R.K. Bolotnova, Wide-Range Equation of State for Water and Steam: Simplified version, High Temperature 49 (2), 303 (2011)

[59] V.A. Khokhlov et al., Complex superelastic and plastic shock wave, caused by a short laser pulse, Proceedings of the Kabardino-Balkarian State University 4 (1), 53 (2014)

[60] N.A. Inogamov et al., Two-temperature hydrodynamic expansion and coupling of strong elastic shock with supersonic melting front produced by ultrashort laser pulse, J. Phys.: Conf. Ser. 500 (05), 192023 (2014) 